\title{
Overview of Millimeter Wave Communications for Fifth-Generation (5G) Wireless Networks-with a focus on Propagation Models
}

\author{
Theodore S. Rappaport, Fellow, IEEE, Yunchou Xing, Student Member, IEEE, George R. MacCartney, Jr., Student \\ Member, IEEE, Andreas F. Molisch, Fellow, IEEE, Evangelos Mellios, Member, IEEE, Jianhua Zhang, Senior Member, \\ IEEE,
}

\begin{abstract}
This paper provides an overview of the features of fifth generation (5G) wireless communication systems now being developed for use in the millimeter wave (mmWave) frequency bands. Early results and key concepts of $5 \mathrm{G}$ networks are presented, and the channel modeling efforts of many international groups for both licensed and unlicensed applications are described here. Propagation parameters and channel models for understanding mmWave propagation, such as line-of-sight (LOS) probabilities, large-scale path loss, and building penetration loss, as modeled by various standardization bodies, are compared over the 0.5-100 GHz range.
\end{abstract}

Index Terms-mmWave; 5G; propagation; cellular network; path loss; channel modeling; channel model standards;

○

\section{INTRODUCTION}

Wireless data traffic has been increasing at a rate of over 50\% er year per subscriber, and this trend is expected to accelerate over the next decade with the continual use of video and the rise of the Internet-of-Things (IoT) [1], [2]. To address this demand, the wireless industry is moving to its fifth generation (5G) of cellular technology that will use millimeter wave (mmWave) frequencies to offer unprecedented spectrum and multi-Gigabit-per-second (Gbps) ndata rates to a mobile device [3]. Mobile devices such as cell bhones are typically referred to as user equipment (UE). A simple analysis illustrated that $1 \mathrm{GHz}$ wide channels at 28 or $73 \mathrm{GHz}$ could offer several Gbps of data rate to UE with modest phased array antennas at the mobile handset [4], and early work showed 15 Gbps peak rates are possible with $4 \times 4$ phased arrays antenna at the UE and $200 \mathrm{~m}$ spacing between base stations (BSs) [5], [6]. Promising studies such as these led the US Federal Communications Commission (FCC) to authorize its 2016 "Spectrum Frontiers" allocation of $10.85 \mathrm{GHz}$ of millimeter wave spectrum for 5 G advancements [7], and several studies [8]-[11] have proposed new mobile radio concepts to support $5 \mathrm{G}$ mobile networks.

T. S. Rappaport (email: tsr@nyu.edu), Y. Xing (email: yx775@nyu.edu), G. R. MacCartney , Jr. (email: gmac@nyu.edu), are with NYU WIRELESS Research Center, and are supported in part by the NYU WIRELESS Industrial Affiliates: AT\&T, CableLabs, Cablevision, Ericsson, Huawei, Intel Corporation, InterDigital Inc., Keysight Technologies, L3 Communications, Nokia, National Instruments, Qualcomm Technologies, SiBeam, Straight Path Communications, OPPO, Sprint, Verizon and UMC, in part by the GAANN Fellowship Program, and in part by the National Science Foundation under Grant 1320472, Grant 1237821, and Grant 1302336. NYU Tandon School of Engineering, 9th Floor, 2 MetroTech Center, Brooklyn, NY 11201.

A. F. Molisch (email: molisch@usc.edu), is with the Ming Hsieh Department of Electrical Engineering, University of Southern California, Los Angeles, CA 90089. His work is supported by the National Science Foundation and Samsung.

E. Mellios (email: Evangelos.Mellios@bristol.ac.uk ), is with the Communication Systems \& Networks Group, University of Bristol, Merchant Venturers Building, Woodland Road, BS8 1UB, Bristol, United Kingdom

J. Zhang (email:jhzhang@bupt.edu.cn), is with State Key Lab of Networking and Switching Technology, Beijing University of Posts and Telecommunications, Mailbox NO.92, 100876.

The authors thank Shu Sun of NYU for her suggestions on this paper.
5G mmWave wireless channel bandwidths will be more than ten times greater than today's $4 \mathrm{G}$ Long-Term Evolution (LTE) $20 \mathrm{MHz}$ cellular channels. Since the wavelengths shrink by an order of magnitude at mmWave when compared to today's $4 \mathrm{G}$ microwave frequencies, diffraction and material penetration will incur greater attenuation, thus elevating the importance of lineof-sight (LOS) propagation, reflection, and scattering. Accurate propagation models are vital for the design of new mmWave signaling protocols (e.g., air interfaces). Over the past few years, measurements and models for a vast array of scenarios have been presented by many companies and research groups [3], [4], [12]][32].

This invited overview paper is organized as follows: Section III summarizes key 5G system concepts of emerging mmWave wireless communication networks and Section III presents 5G propagation challenges and antenna technologies. Section IV] gives a thorough compilation and comparison of recent mmWave channel models developed by various groups and standard bodies, while Section $\mathrm{V}$ provides concluding remarks.

\section{5G S YSTEM CONCEPTS AND AIR INTERFACES}

$5 \mathrm{G}$ promises great flexibility to support a myriad of Internet Protocol (IP) devices, small cell architectures, and dense coverage areas. Applications envisioned for 5G include the Tactile Internet [33], vehicle-to-vehicle communication [34], vehicle-toinfrastructure communication [35], as well as peer-to-peer and machine-to-machine communication [36], all which will require extremely low network latency and on-call demand for large bursts of data over minuscule time epochs [37]. Current 4G LTE and WiFi roundtrip latencies are about 20-60 ms [38], [39], but 5G will offer roundtrip latencies on the order of $1 \mathrm{~ms}$ [40]. As shown in Fig. 1 today's $4 \mathrm{G}$ cellular network is evolving to support $5 \mathrm{G}$, where WiFi off-loading, small cells, and distribution of wideband data will rely on servers at the edges of the network (edge servers) to enable new use cases with lower latency.

\section{A. Backhaul and Fronthaul}

Fig. 1 shows how backhaul connects the fixed cellular infrastructure (e.g., BSs) to the core telephone network and the Internet. Backhaul carries traffic between the local subnetwork (e.g., the connections between UE and BSs) and the core network (e.g., the Internet and the Mobile Switching Telephone Office). 4G and WiFi backhaul, and not the air interface, are often sources of traffic bottlenecks in modern networks since backhaul connections provided by packet-based Ethernet-over-Fiber links typically provide only about 1 Gbps [41], which may be easily consumed by several UEs. In a typical macrocell site, a baseband unit (BBU) is in an enclosure at the base of a remote cell site and is directly connected 


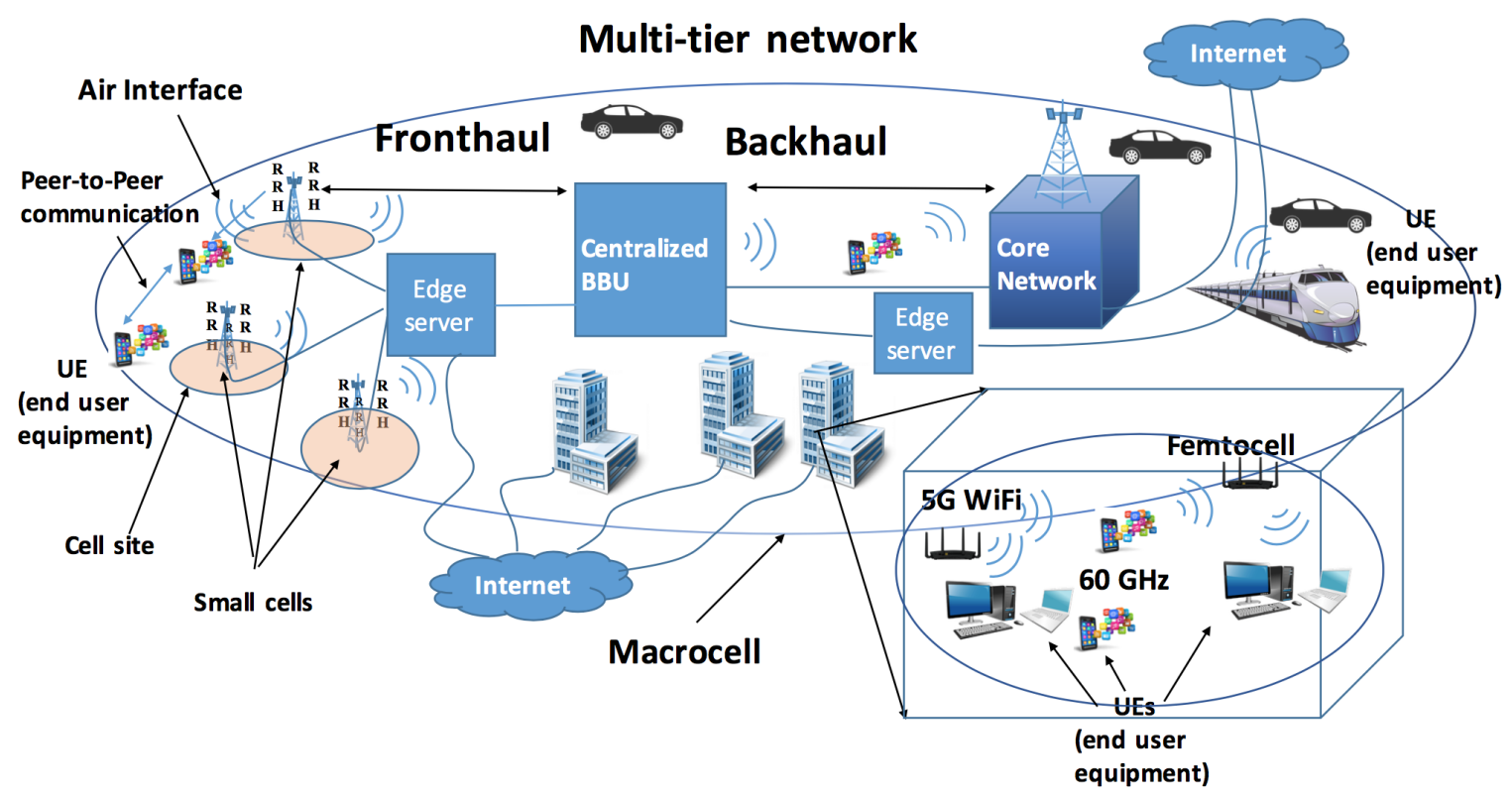

Fig. 1: Mobile networks are evolving from 4G towards 5G. Shown here are small cells, edge servers, wireless backhaul, and 5G multi-tier architecture.

to the backhaul. The BBU processes and modulates IP packet data from the core network into digital baseband signals where they are transmitted to remote radio heads (RRHs). The digital baseband signal travels from the BBU to a RRH via a common public radio interface (CPRI) through a digital radio-over-fiber (DRoF) connection, also known as fronthaul. The RRH converts the digital signal to analog for transmission over the air at the carrier frequency by connecting to amplifiers and antennas to transmit the downlink from the cell tower. The RRH also converts the received radio frequency $(\mathrm{RF})$ uplink signal from the UEs into a digital baseband signal which travels from the RRH to the BBU via the same CPRI and D-RoF connection to the base of the cell tower. The BBU then processes and packetizes the digital baseband signal from the RRH and sends it through a backhaul connection to the core network. In summary, fronthaul is the connection between the $\mathrm{RRH}$ and BBU in both directions and backhaul is the connection between the BBU and the core network in both directions.

Modern cellular architectures support a more flexible deployment of radio resources that may be distributed using a cloud radio access network technique, where a BS is split into two parts [42], one part where the RRHs are at remote cell sites, and in the other part, one centralized BBU is located up to tens of kilometers away (see Fig. 1). CPRI is used for fronthaul, and interconnects the centralized BBU and multiple RRHs through D-RoF. MmWave wireless backhaul and fronthaul will offer fiber-like data rates and bandwidth to infrastructure without the expense of deploying wired backhaul networks or long-range D-RoF [9], [43], [44].

\section{B. Small Cells}

An effective way to increase area spectral efficiency is to shrink cell size [40], [45], [46] where the reduced number of users per cell, caused by cell shrinking, provides more spectrum to each user. Total network capacity vastly increases by shrinking cells and reusing the spectrum, and future nomadic BSs and direct deviceto-device connections between UEs are envisioned to emerge in $5 \mathrm{G}$ for even greater capacity per user [47]. Femtocells that can dynamically change their connection to the operator's core network will face challenges such as managing RF interference and keeping timing and synchronization, and various interference avoidance and adaptive power control strategies have been suggested [45]. An analysis of the wireless backhaul traffic at $5.8 \mathrm{GHz}, 28 \mathrm{GHz}$, and $60 \mathrm{GHz}$ in two typical network architectures showed that spectral efficiency and energy efficiency increased as the number of small cells increased [48], and backhaul measurements and models at $73 \mathrm{GHz}$ were made in New York City [20], [49]. Work in [50] showed a theory for power consumption analysis, which is strikingly similar to noise figure, for comparing energy efficiency and power consumption in wideband networks. An early small-cell paper [51] gave insights into enhancing user throughput, reducing signaling overhead, and reducing dropped call likelihoods.

\section{Multi-tier Architecture}

The roadmap for $5 \mathrm{G}$ networks will exploit a multi-tier architecture of larger coverage $4 \mathrm{G}$ cells with an underlying network of closer-spaced 5G BSs as shown in Fig. 1. A multi-tier architecture allows users in different tiers to have different priorities for channel access and different kinds of connections (e.g., macrocells, small cells, and device-to-device connections), thus supporting higher data rates, lower latencies, optimized energy consumption, and interference management by using resource-aware criteria for the BS association and traffic loads allocated over time and space [52]. Schemes and models for load balanced heterogeneous networks in a multi-tier architecture are given in [53], [54]. 5G applications will also require novel network architectures that support the convergence of different wireless technologies (e.g., WiFi, LTE, mmWave, low-power IoT) that will interact in a flexible and seamless manner using Software Defined Networking and Network Virtualization principles [55], [56].

\section{D. $5 G$ Air Interface}

The design of new physical layer air interfaces is an active area of $5 \mathrm{G}$ research. Signaling schemes that provide lower latency, rapid beamforming and synchronization, with much smaller time slots and better spectral efficiency than the orthogonal frequency division 
multiplexing (OFDM) used in 4G, will emerge. A novel modulation that exploits the dead time in the single-carrier frequency domain modulation method used in today's 4G LTE uplink is given in [5]. Work in [10] reviews linear modulation schemes such as filter bank multicarrier (FBMC) modulation wherein subcarriers are passed through filters that suppress sidelobes. Generalized frequency division multiplexing (GFDM) is proposed in [11], where it is shown that, when compared with OFDM used in current 4G LTE (which has one cyclic prefix per symbol and high outof-band emissions [57]), GFDM improves the spectral efficiency and has approximately $15 \mathrm{~dB}$ weaker out-of-band emissions. Orthogonal time-frequency-space (OTFS) modulation that spreads the signals in the time-frequency plane has also been suggested, due to superior diversity and higher flexibility in pilot design [58]. Channel state feedback and management to support directional beam search/steering will also be vital [59], [60].

\section{E. $5 G$ Unlicensed WiFi}

MmWave WiFi for the 57-64 GHz unlicensed bands has been in development for nearly a decade, with the WirelessHD and IEEE 802.11ad standardization process beginning in 2007, and 2009, respectively [61]. IEEE 802.11ad devices, which can reach 7 Gbps peak rates [62], and WirelessHD products which can reach 4 Gbps with theoretical data rates as high as $25 \mathrm{Gbps}$ [63], are both already available in the market. Building on the history of WiFi standard IEEE 802.11n [64], [65], two newer standards, IEEE 802.11ac and 802.11ad, are amendments that improve the throughput to reach $1 \mathrm{Gbps}$ in the $5 \mathrm{GHz}$ band and up to $7 \mathrm{Gbps}$ in the $60 \mathrm{GHz}$ band, respectively. An overview of IEEE Gigabit wireless local area network (WLAN) amendments (IEEE 802.11ac and 802.11ad) [66]-[68] shows the suitability of these two standards for multigigabit communications. For the 802.11ad standard [69], notable features include fast session transfer for seamless data rate fall back (and rate rise) between $60 \mathrm{GHz}$ and $2.4 / 5 \mathrm{GHz}$ PHYs, and media access control (MAC) enhancements for directional antennas, beamforming, backhaul, relays and spatial reuse techniques. For enhancements of the PHY layer, beamforming using directional antennas or antenna arrays is used to overcome the increased loss at $60 \mathrm{GHz}$ [61]. IEEE 802.11ay standard is an ongoing project with the goal to support a maximum throughput of at least $20 \mathrm{Gbps}$ in the $60 \mathrm{GHz}$ unlicensed band [70]. Newer WiFi standards are sure to emerge to exploit the new $64-71 \mathrm{GHz}$ unlicensed spectrum in the US [7].

\section{F. Vehicular Networks}

Vehicle-to-vehicle $(\mathrm{V} 2 \mathrm{~V})$ communications are an important tool for increasing road safety and reducing traffic congestion. Currently the most investigated system is the IEEE $802.11 \mathrm{p}$ standard which works in $5.9 \mathrm{GHz}$ band for $\mathrm{V} 2 \mathrm{~V}$ and vehicle-to-infrastructure (V2I) communication, and is known as dedicated short-range communications (DSRC) [71]. The mmWave bands (e.g., $24 \mathrm{GHz}$ and 77 $\mathrm{GHz}[7]$ ) are attractive for V2V and V2I, (e.g., cars, high-speed railway and subway systems) since connected vehicles will need Gbps date rates, which cannot be achieved in the $10 \mathrm{MHz}$ channel bandwidths at $5.9 \mathrm{GHz}$ in current $4 \mathrm{G}[72]-[74]$. Limitations of $\mathrm{V} 2 \mathrm{~V}$ connectivity include the difficulty in achieving realistic spatial consistency to sustain the data-link connection for high-speed mobility vehicles [12], [75]. Evaluations have shown that narrow beam directional antennas are more suitable for IEEE 802.11pbased systems [76], and several schemes aimed at utilizing adaptive

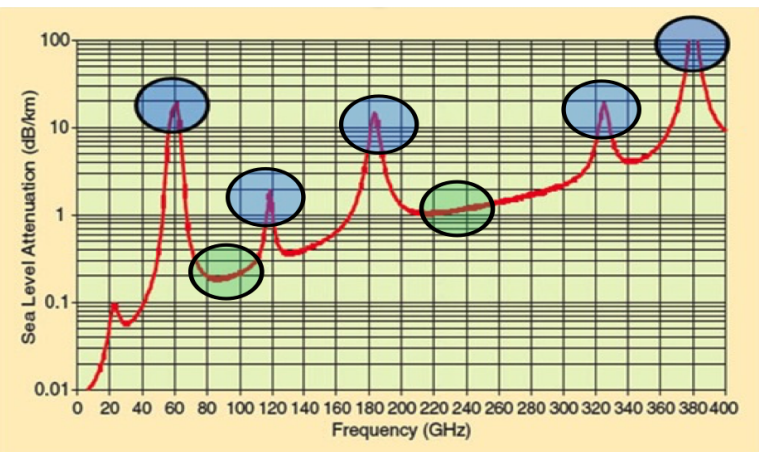

Fig. 2: Atmospheric absorption of electromagnetic waves at sea level versus frequency, showing the additional path loss due to atmospheric absorption [78].

antennas for fast moving $\mathrm{V} 2 \mathrm{~V}$ communications are provided in [77].

\section{5G Antenna and PRopagation Challenges}

The entire radio spectrum up to $5.8 \mathrm{GHz}$ that has been used for global wireless communications throughout the past 100 years easily fits within the bandwidth of the single $60 \mathrm{GHz}$ unlicensed band, yet there is so much more spectrum still available above $60 \mathrm{GHz}$ [4], [7], [61], as shown in Figure C.1 on page 40 of [61]. With radio frequency integrated circuits (RFIC) now routinely manufactured for 24 and $77 \mathrm{GHz}$ vehicular radar, and IEEE 802.11ad WiGig devices now becoming mainstream in high-end laptops and cellphones, low-cost electronics will be viable for the evolution of massively broadband $5 \mathrm{G}$ millimeter wave communications [78].

Today, most spectrum above $30 \mathrm{GHz}$ is used for military applications or deep-space astronomy reception, but the recent FCC Spectrum Frontiers ruling has assigned many bands for mobile and backhaul communications. The various resonances of oxygen and other gasses in air, however, cause certain bands to suffer from signal absorption in the atmosphere. Fig. 2 illustrates how the bands of $183 \mathrm{GHz}, 325 \mathrm{GHz}$, and especially $380 \mathrm{GHz}$ suffer much greater attenuation over distance due to the molecular resonances of various components of the atmosphere, beyond the natural Friis' free space loss, making these particular bands well suited for very close-in communications and "whisper radio" applications where massive bandwidth channels will attenuate very rapidly out to a few meters or fractions of a meter [3], [61]. Fig. 2] also shows many mmWave bands only suffer $1-2 \mathrm{~dB}$ more loss than caused by free space propagation per $\mathrm{km}$ in air [79], [80]. Rain and hail cause substantial attenuation at frequencies above $10 \mathrm{GHz}$ [81], and $73 \mathrm{GHz}$ signals attenuate at $10 \mathrm{~dB} / \mathrm{km}$ for a $50 \mathrm{~mm} / \mathrm{hr}$ rain rate [3], [61], [82]. Interestingly, as shown in [3], [78] rain attenuation flattens out at $100 \mathrm{GHz}$ to $500 \mathrm{GHz}$, and for all mmWave frequencies, rain or snow attenuation may be overcome with additional antenna gain or transmit power. Also, the size and orientation of rain drops and clouds may determine the particular amount of attenuation on air-to-ground links such that satellites could undergo more localized and perhaps less rain attenuation than terrestrial links at mmWave frequencies.

While it is commonly believed that path loss increases dramatically by moving up to mmWave frequencies, extensive work in various environments in [21], [28], [29], [83], [84] shows that Friis' equation [85] dictates this is true only when the antenna gain is assumed to be constant over frequency. If the physical size of the antenna (e.g., effective aperture) is kept constant over 
frequency at both link ends and the weather is clear, then path loss in free space actually decreases quadratically as frequency increases [61]. The larger antenna gains at higher frequencies require adaptive beam steering for general use at both the BS and UE, compared to legacy mobile antennas with lower gain [61]. Beam steerable antenna technologies estimate directions of arrival and adaptively switch beam patterns to mitigate interference and to capture the signal of interest. Adaptive arrays are essential for mmWave communications to compensate the path loss caused by blockage from dynamic obstacles [29], [61], [80], [86]-[88].

Penetration into buildings may pose a significant challenge for mmWave communication, and this is a distinct difference from today's UHF/microwave systems. Measurements at $38 \mathrm{GHz}$ described in [89] found a penetration loss of nearly $25 \mathrm{~dB}$ for a tinted glass window and $37 \mathrm{~dB}$ for a glass door. Measurements at $28 \mathrm{GHz}[3]$ showed that outdoor tinted glass and brick pillars had penetration losses of $40.1 \mathrm{~dB}$ and $28.3 \mathrm{~dB}$, respectively, but indoor clear glass and drywall only had $3.6 \mathrm{~dB}$ and $6.8 \mathrm{~dB}$ of loss. Work in [90] shows penetration losses for many common materials and provides normalized attenuation (e.g., in $\mathrm{dB} / \mathrm{cm}$ ) at $73 \mathrm{GHz}$.

MmWave will need to exploit and rapidly adapt to the spatial dynamics of the wireless channel since greater gain antennas will be used to overcome path loss. Diffuse scattering from rough surfaces may introduce large signal variations over very short travel distances (just a few centimeters) as shown in Fig. 3 Such rapid variations of the channel must be anticipated for proper design of channel state feedback algorithms, link adaptation schemes and beam-forming/tracking algorithms, as well as ensuring efficient design of MAC and Network layer transmission control protocols (TCP) that induce re-transmissions. Measurement of diffuse scattering at $60 \mathrm{GHz}$ on several rough and smooth wall surfaces [91], [92] demonstrated large signal level variations in the first order specular and in the non-specular scattered components (with fade depths of up to $20 \mathrm{~dB}$ ) as a user moved by a few centimeters. In addition, the existence of multipath from nearly co-incident signals can create severe small-scale variations in the channel frequency response. As reported in [91], [92], measurements showed that reflection from rough materials might suffer from high depolarization, a phenomenon that highlights the need for further investigation into the potential benefits of exploiting polarization diversity for the performance enhancement of mmWave communication systems. Work in [93] showed shallow Ricean fading of multipath components and exponential decaying trends for spatial autocorrelation at 28 $\mathrm{GHz}$ and quick decorrelation at about 2.5 wavelengths for the LOS environment. Work in [75] shows that received power of wideband $73 \mathrm{GHz}$ signals has a stationary mean over slight movements but average power can change by $25 \mathrm{~dB}$ as the mobile transitioned a building cornor from non-line-of-sight (NLOS) to LOS in an urban microcell (UMi) environment [88], [94]. Measurements at 10, 20 and $26 \mathrm{GHz}$ demonstrate that diffraction loss can be predicted using well-known models as a mobile moves around a corner using directional antennas [95], and human body blockage causes more than $40 \mathrm{~dB}$ of fading [88], [94].

It is not obvious that the stationarity region size or small-scale statistics derived from 3GPP TR 36.873 [96] and other sub-6 GHz channel models, or those used by 3GPP or ITU above $6 \mathrm{GHz}$ are valid for mmWave channels [80], [97]-[100]. Recent measurements [75], [91], [94] indicate very sharp spatial decorrelation over small distance movements of just a few tens of wavelengths at mmWave, depending on antenna orientation, but more work is needed in this area. The necessity and proper form of spatial consistency,
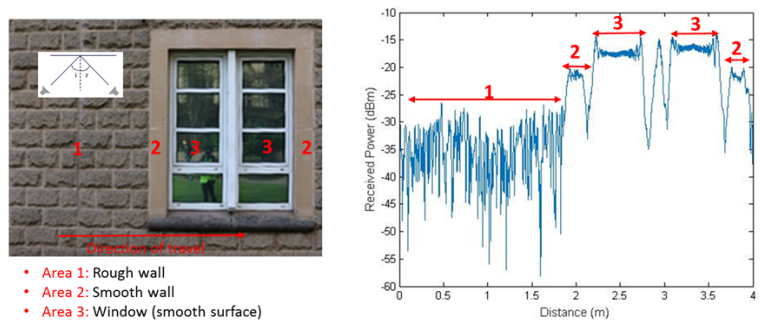

Fig. 3: Results of diffuse scattering measurements at $60 \mathrm{GHz}$, where smooth surfaces (e.g., windows) offer high correlation over distance, but signals from rough surfaces seem less correlated over distance [91], [92].

if borne out by measurements, have yet to be fully understood by the research community.

\section{Channel Modeling}

Channel models are required for simulating propagation in a reproducible and cost-effective way, and are used to accurately design and compare radio air interfaces and system deployment. Common wireless channel model parameters include carrier frequency, bandwidth, 2-D or 3-D distance between transmitter (TX) and receiver (RX), environmental effects, and other requirements needed to build globally standardized equipment and systems. The definitive challenge for a $5 \mathrm{G}$ channel model is to provide a fundamental physical basis, while being flexible, and accurate, especially across a wide frequency range such as $0.5 \mathrm{GHz}$ to 100 GHz. Recently, a great deal of research aimed at understanding the propagation mechanisms and channel behavior at the frequencies above $6 \mathrm{GHz}$ has been published [3], [4], [12]-[32], [40], [60], [73], [75], [78], [81], [83], [84], [89]-[95], [101]-[111]. The specific types of antennas used and numbers of measurements collected vary widely and may generally be found in the referenced work.

For the remainder of this paper, the models for LOS probability, path loss, and building penetration introduced by four major organizations in the past years are reviewed and compared: (i) the 3rd Generation Partnership Project (3GPP TR 38.901 [101]), which attempts to provide channel models from $0.5-100 \mathrm{GHz}$ based on a modification of 3GPP's extensive effort to develop models from 6 to $100 \mathrm{GHz}$ in TR 38.900 [112]. 3GPP TR documents are a continual work in progress and serve as the international industry standard for 5G cellular, (ii) 5G Channel Model (5GCM) [12], an ad-hoc group of 15 companies and universities that developed models based on extensive measurement campaigns and helped seed 3GPP understanding for TR 38.900 [112], (iii) Mobile and wireless communications Enablers for the Twenty-twenty Information Society (METIS) [102] a large research project sponsored by European Union, and (iv) Millimeter-Wave Based Mobile Radio Access Network for Fifth Generation Integrated Communications (mmMAGIC) [92], another large research project sponsored by the European Union. While many of the participants overlap in these standards bodies, the final models between those groups are somewhat distinct. It is important to note that recent work has found discrepancies between standardized models and measured results [29], [99], [100].

\section{A. LOS Probability Model}

The mobile industry has found benefit in describing path loss for both LOS and NLOS conditions separately. As a consequence, 
TABLE I: LOS probability models in the UMi scenario.

\begin{tabular}{|c|c|c|}
\hline & LOS probability models (distances are in meters) & Parameters \\
\hline 3GPP TR 38.901 |101] & $\begin{array}{l}\text { Outdoor users: } \\
P_{L O S}\left(d_{2 D}\right)=\min \left(d_{1} / d_{2 D}, 1\right)\left(1-\exp \left(-d_{2 D} / d_{2}\right)\right)+\exp \left(-d_{2 D} / d_{2}\right) \\
\text { Indoor users: } \\
\text { Use } d_{2 D-\text { out }} \text { in the formula above instead of } d_{2 D}\end{array}$ & $\begin{array}{l}d_{1}=18 \mathrm{~m} \\
d_{2}=36 \mathrm{~m}\end{array}$ \\
\hline $5 \mathrm{GCM}[12$ & $\begin{array}{l}\boldsymbol{d}_{\mathbf{1}} / \boldsymbol{d}_{\mathbf{2}} \text { model: } \\
P_{L O S}\left(d_{2 D}\right)=\min \left(d_{1} / d_{2 D}, 1\right)\left(1-\exp \left(-d_{2 D} / d_{2}\right)\right)+\exp \left(-d_{2 D} / d_{2}\right) \\
\text { NYU (squared) model: } \\
P_{L O S}\left(d_{2 D}\right)=\left(\min \left(d_{1} / d_{2 D}, 1\right)\left(1-\exp \left(-d_{2 D} / d_{2}\right)\right)+\exp \left(-d_{2 D} / d_{2}\right)\right)^{2}\end{array}$ & $\begin{array}{c}\boldsymbol{d}_{\mathbf{1}} / \boldsymbol{d}_{\mathbf{2}} \text { model: } \\
d_{1}=20 \mathrm{~m} \\
d_{2}=39 \mathrm{~m} \\
\text { NYU (squared) model: } \\
d_{1}=22 \mathrm{~m} \\
d_{2}=100 \mathrm{~m} \\
\end{array}$ \\
\hline METIS 102 & $\begin{array}{l}\text { Outdoor users: } \\
P_{L O S}\left(d_{2 D}\right)=\min \left(d_{1} / d_{2 D}, 1\right)\left(1-\exp \left(-d_{2 D} / d_{2}\right)\right)+\exp \left(-d_{2 D} / d_{2}\right) \\
\text { Indoor users: } \\
\text { Use } d_{2 D-\text { out }} \text { in the formula above instead of } d_{2 D}\end{array}$ & $\begin{aligned} d_{1} & =18 \mathrm{~m} \\
d_{2} & =36 \mathrm{~m} \\
10 \mathrm{~m} & \leq d_{2 D}\end{aligned}$ \\
\hline mmMAGIC 92 & $\begin{array}{l}\text { Outdoor users: } \\
P\left(d_{2 D}\right)=\min \left(d_{1} / d_{2 D}, 1\right)\left(1-\exp \left(-d_{2 D} / d_{2}\right)\right)+\exp \left(-d_{2 D} / d_{2}\right) \\
\text { Indoor users: } \\
\text { Use } d_{2 D-\text { out }} \text { in the formula above instead of } d_{2 D}\end{array}$ & $\begin{array}{l}d_{1}=20 \mathrm{~m} \\
d_{2}=39 \mathrm{~m}\end{array}$ \\
\hline
\end{tabular}

TABLE II: LOS probability models for the UMa scenario

\begin{tabular}{|c|c|c|}
\hline & LOS probability models (distances are in meters) & Parameters \\
\hline 3GPP TR 38.901 [101] & 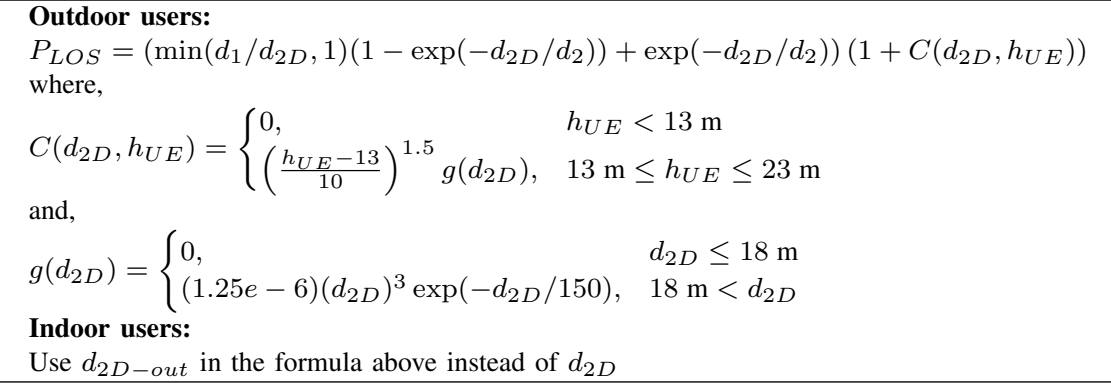 & $\begin{array}{l}d_{1}=18 \mathrm{~m} \\
d_{2}=63 \mathrm{~m}\end{array}$ \\
\hline $5 \mathrm{GCM}[12]$ & $\begin{array}{l}\boldsymbol{d}_{\mathbf{1}} / \boldsymbol{d}_{\mathbf{2}} \text { model: } \\
P_{L O S}=\left(\min \left(d_{1} / d_{2 D}, 1\right)\left(1-\exp \left(-d_{2 D} / d_{2}\right)\right)+\exp \left(-d_{2 D} / d_{2}\right)\right)\left(1+C\left(d_{2 D}, h_{U E}\right)\right) \\
\text { NYU (squared) model: } \\
P_{L O S}=\left(\left(\min \left(d_{1} / d_{2 D}, 1\right)\left(1-\exp \left(-d_{2 D} / d_{2}\right)\right)+\exp \left(-d_{2 D} / d_{2}\right)\right)\left(1+C\left(d_{2 D}, h_{U E}\right)\right)\right)^{2}\end{array}$ & $\begin{array}{c}\boldsymbol{d}_{\mathbf{1}} / \boldsymbol{d}_{\mathbf{2}} \text { model: } \\
d_{1}=20 \mathrm{~m} \\
d_{2}=66 \mathrm{~m} \\
\text { NYU (squared) model: } \\
d_{1}=20 \mathrm{~m} \\
d_{2}=160 \mathrm{~m}\end{array}$ \\
\hline METIS 102 & $\begin{array}{l}\text { Outdoor users: } \\
P_{L O S}=\left(\min \left(d_{1} / d_{2 D}, 1\right)\left(1-\exp \left(-d_{2 D} / d_{2}\right)\right)+\exp \left(-d_{2 D} / d_{2}\right)\right)\left(1+C\left(d_{2 D}, h_{U E}\right)\right) \\
\text { Indoor users: } \\
\text { Use } d_{2 D-\text { out }} \text { in the formula above instead of } d_{2 D}\end{array}$ & $\begin{array}{l}d_{1}=18 \mathrm{~m} \\
d_{2}=63 \mathrm{~m}\end{array}$ \\
\hline
\end{tabular}

models for the probability of LOS are required, i.e., statistical models are needed to predict the likelihood that a UE is within a clear LOS of the BS, or in an NLOS region due to obstructions. LOS propagation will offer more reliable performance in mmWave communications as compared to NLOS conditions, given the greater diffraction loss at higher frequencies compared to sub-6 $\mathrm{GHz}$ bands where diffraction is a dominant propagation mechanism [75], [95], and given the larger path loss exponent as well as increased shadowing variance in NLOS as compared to LOS [28]. The LOS probability is modeled as a function of the 2D TX-RX (T-R) separation distance and is frequency-independent, as it is solely based on the geometry and layout of an environment or scenario [23]. In the approach of 5GCM [12], the LOS state is determined by a map-based approach in which only the TX and the RX positions are considered for determining if the direct path between the TX and RX is blocked.

1) UMi LOS Probability: The UMi scenarios include high user density open areas and street canyons with BS heights below rooftops (e.g., 3-20 m), UE heights at ground level (e.g., $1.5 \mathrm{~m}$ ) and inter-site distances (ISDs) of $200 \mathrm{~m}$ or less [96], [106]. The UMi LOS probability models developed by the various parties are provided in Table $\mathrm{I}$ and are detailed below.

a) 3GPP TR 38.901: The antenna height is assumed to be $10 \mathrm{~m}$ in the UMi LOS probability model [101] and the model is referred to as the 3GPP/ITU $d_{1} / d_{2}$ model (it originates in [96], [106]), with $d_{1}$ and $d_{2}$ curve-fit parameters shown in Table I] In [101], model parameters were found to be $d_{1}=18 \mathrm{~m}$ and $d_{2}=$ $36 \mathrm{~m}$ for UMi. For a link between an outdoor BS and an indoor UE, the model uses the outdoor distance $d_{2 D-o u t}$, which is the distance from the BS to the surface of the indoor building, to replace $d_{2 D}$.

b) 5GCM: 5GCM provides two LOS probability models, the first one is identical in form to the 3GPP TR 38.901 outdoor model [101], but with slightly different curve-fit parameters $\left(d_{1}\right.$ and $\left.d_{2}\right)$. The second LOS probability model is the NYU squared model [23], which improves the accuracy of the $d_{1} / d_{2}$ model by including a square on the last term. The NYU model was developed using a much finer resolution intersection test than used by 3GPP TR 38.901, and used a real-world database in downtown New York City [23]. For UMi, the 5GCM $d_{1} / d_{2}$ model has a slightly smaller mean square error (MSE), but the NYU squared model has a more realistic and rapid decay over distance for urban clutter [12], [23]. 


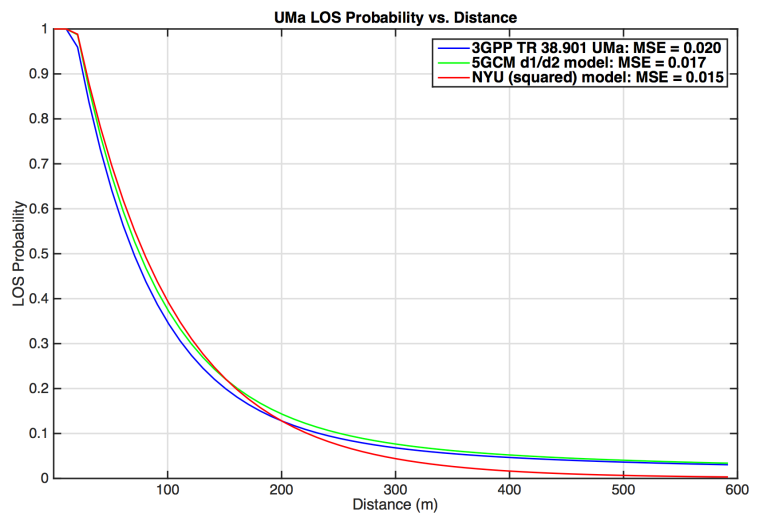

Fig. 4: Comparison among three different LOS probability models in UMa scenario.

c) METIS: The LOS probability model used in METIS [102] is based on the work of 3GPP TR 36.873 [96], and has the same form and the same parameter values as the 3GPP TR 38.901 model in Table I where the minimum T-R separation distance is assumed to be $10 \mathrm{~m}$ in the UMi scenario.

d) mmMAGIC: For the UMi scenario, the mmMAGIC LOS probability model and parameter values are identical to the 5GCM $d_{1} / d_{2}$ model [12].

2) UMa LOS Probability: Urban macrocell (UMa) scenarios typically have BSs mounted above rooftop levels of surrounding buildings (e.g., 25-30 m) with UE heights at ground level (e.g., $1.5 \mathrm{~m}$ ) and ISDs no more than $500 \mathrm{~m}$ [96], [106]. The UMa LOS probability models are given in Table $\overline{\mathrm{II}}$ and are identical to the UMi LOS probability models but with different $d_{1}$ and $d_{2}$ values.

a) 3GPP TR 38.901: The 3GPP TR 38.901 UMa LOS probability models for outdoor and indoor users are presented in Table II, where for indoor users, $d_{2 D-o u t}$ is used instead of $d_{2 D}$ and the models are derived assuming the TX antenna height is 25 $\mathrm{m}$. Due to the larger antenna heights in the UMa scenario, mobile height is an added parameter of the LOS probability as shown in Table II where $h_{U E}$ represents the UE antenna height above ground.

b) 5GCM: The UMa LOS probability models in the 5GCM white paper [12] are of the same form as those in 3GPP TR 38.901 [101], but with different $d_{1}$ and $d_{2}$ values. The 5GCM includes the NYU squared option [23], similar to the UMi scenario. Differences between the 3GPP TR 38.901 and 5GCM UMa LOS probability models are given via MSE in Fig. 4 for a UE height of $1.5 \mathrm{~m}$. Similar performances are found among the three models, with the NYU squared model having the lowest MSE, while also providing the most conservative (e.g., lowest probability) for LOS at distance of several hundred meters [12], [23].

c) METIS: The LOS probability model used in [102] has the same form as the one in 3GPP TR 38.901 in Table III and the minimum T-R separation distance is assumed to be $35 \mathrm{~m}$ in the UMa scenario.

d) mmMAGIC: The UMa scenario is taken into account in the channel model, however, it is not explicitly mentioned in the table since frequency spectrum above $6 \mathrm{GHz}$ is expected to be used for small cell BSs.

3) InH LOS Probability:

a) 3GPP TR 38.901: The indoor office environment consists of two types: indoor hotspot ( $\mathrm{InH})$-Mixed office and $\mathrm{InH}-\mathrm{Open}$ office, where the density of obstructions is greater in the mixed office. LOS probability models for a TX antenna height of $3 \mathrm{~m}$
TABLE III: LOS probability models in the InH scenario

\begin{tabular}{|c|c|c|}
\hline \multicolumn{3}{|c|}{ 3GPP TR 38.901 101 (all distances are in meters) } \\
\hline \multicolumn{3}{|c|}{ InH-Mixed office: } \\
\hline \multicolumn{3}{|c|}{$(1$} \\
\hline$P_{L O S}=\{\epsilon$ & $\begin{array}{l}\exp \left(-\left(d_{2 D}-1.2\right) / 4.7\right) \\
\exp \left(-\left(d_{2 D}-6.5\right) / 32.6\right) \cdot 0.32\end{array}$ & $\begin{array}{l}1.2 \mathrm{~m}<d_{2 D}<6.5 \mathrm{~m} \\
6.5 \mathrm{~m} \leq d_{2 D}\end{array}$ \\
\hline \multicolumn{3}{|c|}{ InH-Open office: } \\
\hline$P_{L O S}^{\text {Open-office }}$ & $=\left\{\begin{array}{l}1, \\
\exp \left(-\left(d_{2 D}-5\right) / 70.8\right) \\
\exp \left(-\left(d_{2 D}-49\right) / 211.7\right) \cdot 0.5\end{array}\right.$ & $\begin{aligned} & d_{2 D} \leq 5 \mathrm{~m} \\
& 5 \mathrm{~m}<d_{2 D}<49 \mathrm{~m} \\
54, & 49 \mathrm{~m} \leq d_{2 D}\end{aligned}$ \\
\hline \multicolumn{3}{|c|}{ 5GCM $[12$} \\
\hline$P_{L O S}=\{$ & $\begin{array}{l}1 \\
\exp \left(-\left(d_{2 D}-1.2\right) / 4.7\right) \\
\exp \left(-\left(d_{2 D}-6.5\right) / 32.6\right) \cdot 0.32\end{array}$ & $\begin{array}{l}d_{2 D} \leq 1.2 \mathrm{~m} \\
1.2 \mathrm{~m}<d_{2 D}<6.5 \mathrm{~m} \\
6.5 \mathrm{~m} \leq d_{2 D}\end{array}$ \\
\hline \multicolumn{3}{|c|}{ mmMAGIC $|92|$} \\
\hline$P_{L O S}=$ & $\left\{\begin{array}{l}1 \\
\exp \left(-\left(d_{2 D}-1.2\right) / 4.7\right) \\
\exp \left(-\left(d_{2 D}-6.5\right) / 32.6\right) \cdot 0.32\end{array}\right.$ & $\begin{array}{l}d_{2 D} \leq 1.2 \mathrm{~m} \\
1.2<d_{2 D}<6.5 \mathrm{~m} \\
6.5 \mathrm{~m} \leq d_{2 D}\end{array}$ \\
\hline
\end{tabular}

for the InH-Mixed office and InH-Open office sub-scenarios are provided in Table III

b) 5GCM: In [12], different types of indoor office environments were investigated, including open-plan offices with cubicle areas, closed-plan offices with corridors and meeting rooms, and hybrid-plan offices with both open and closed areas, and based on ray-tracing simulations [107]. See Table III] and [12].

c) mmMAGIC: mmMAGIC adopted the 5GCM InH scenario LOS probability model [12].

4) RMa LOS Probability: Rural macrocell (RMa) scenarios typically have BS heights that range between $10 \mathrm{~m}$ and $150 \mathrm{~m}$ with UE heights at ground level (e.g., $1.5 \mathrm{~m}$ ) and ISDs up to 5000 m [96], [106]. The LOS probabilities for RMa were not specified in METIS or 5GCM channel models. The 3GPP TR 38.901 [101] RMa LOS probability model was adopted from the International Telecommunications Union-Radio (ITU-R) M.2135 [106], which was derived from the WINNER [113] RMa LOS probability model and is given by:

$$
P_{L O S}= \begin{cases}1, & d_{2 D} \leq 10 \mathrm{~m} \\ \exp \left(-\frac{d_{2 D}-10}{1000}\right), & d_{2 D}>10 \mathrm{~m}\end{cases}
$$

where $P_{L O S}$ is the LOS probability for a specific T-R pair, $d_{2 D}$ is the 2D T-R separation distance (in meters). Similarly, the RMa LOS probability 3GPP TR 38.901 Release 14 channel model [101] is adopted entirely from ITU-R M.2135 [106]. As shown in [19], [24], caution is advised since these models were derived from urban (not rural) scenarios below $6 \mathrm{GHz}$.

\section{B. Large-Scale Path Loss Models}

There are three basic types of large-scale path loss models to predict mmWave signal strength over distance for the vast mmWave frequency range (with antenna gains included in the link budget and not in the slope of path loss as shown in Eq. (3.9) of [61], also see p.3040 in [20]). These include the close-in (CI) free space reference distance model (with a $1 \mathrm{~m}$ reference distance) [20], [28], [83], [84], the CI model with a frequency-weighted or height weighted path loss exponent (CIF and CIH models) [18], [19], [21], [24], and the floating intercept (FI) path loss model, also known as the ABG model because of its use of three parameters $\alpha, \beta$, and $\gamma[18],[20]-[22],[108],[114]$. Standard bodies historically create omnidirectional path loss models with the assumption of unity gain antennas for generality. However, it is worth noting that 
TABLE IV: Omnidirectional Path loss models in the UMi scenario

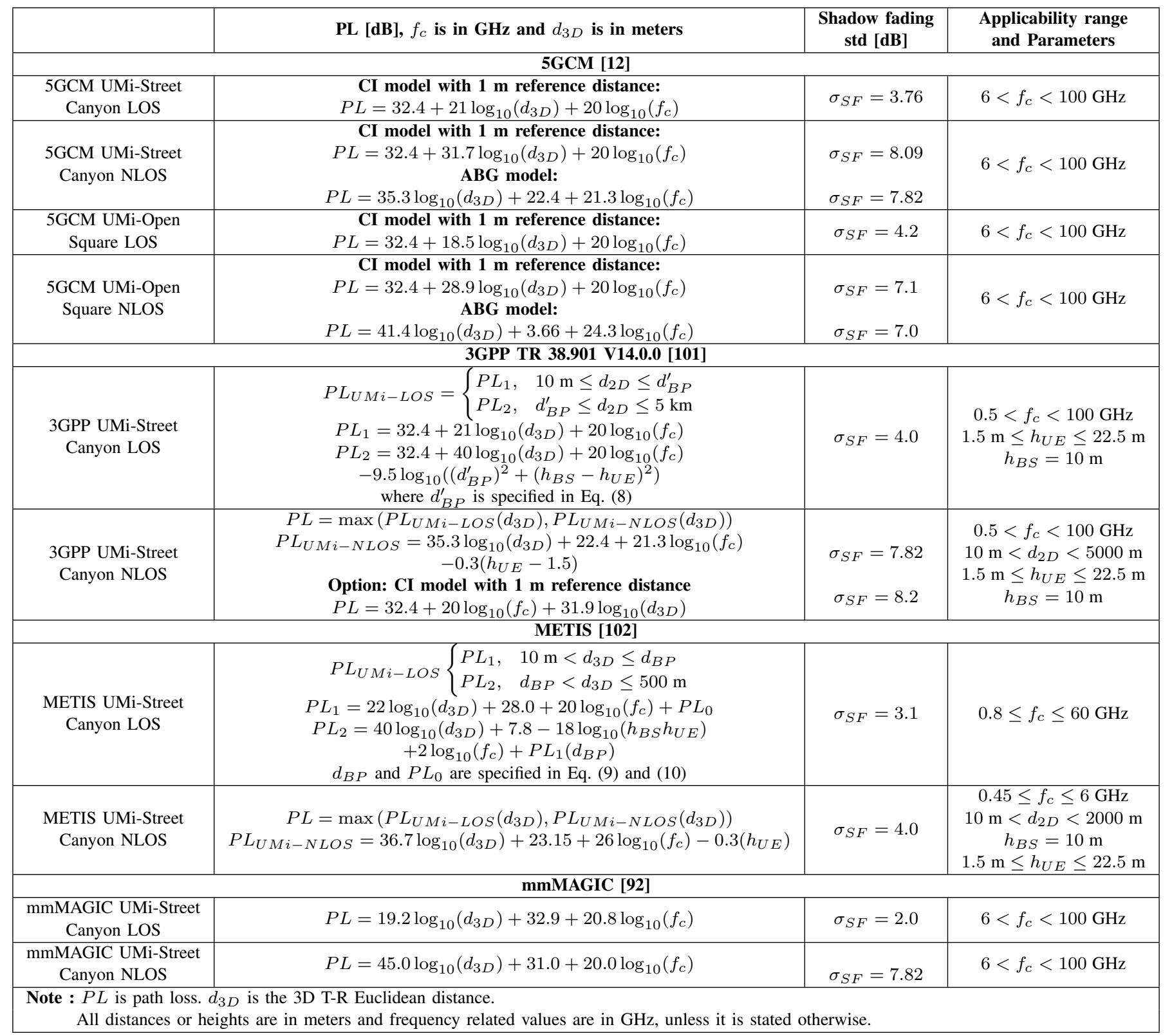

omnidirectional path loss models will not be usable in directional antenna system analysis unless the antenna patterns and true spatial and temporal multipath channel statistics are known or properly modeled [19], [20], [29], [80], [99], [115], [116].

The CI path loss model accounts for the frequency dependency of path loss by using a close-in reference distance based on Friis' law as given by [12], [19], [21], [24], [28]:

$$
P L^{C I}\left(f_{c}, d_{3 D}\right)[\mathrm{dB}]=\operatorname{FSPL}\left(f_{c}, 1 \mathrm{~m}\right)+10 n \log _{10}\left(d_{3 D}\right)+\chi_{\sigma}^{C I}
$$

where $\chi_{\sigma}^{C I}$ is the shadow fading (SF) that is modeled as a zeromean Gaussian random variable with a standard deviation in $\mathrm{dB}$, $n$ is the path loss exponent (PLE) found by minimizing the error of the measured data to 2$), d_{3 D}>1 \mathrm{~m}, \operatorname{FSPL}(f, 1 \mathrm{~m})$ is the free space path loss (FSPL) at frequency $f_{c}$ in $\mathrm{GHz}$ at $1 \mathrm{~m}$ and is calculated by [19], [85]:

$$
\operatorname{FSPL}\left(f_{c}, 1 \mathrm{~m}\right)=20 \log _{10}\left(\frac{4 \pi f_{c} \times 10^{9}}{c}\right)=32.4+20 \log _{10}\left(f_{c}\right)[\mathrm{dB}]
$$

where $c$ is the speed of light, $3 \times 10^{8} \mathrm{~m} / \mathrm{s}$. Using (3) it is clear that (2) can be represented as given in Table IV. The standard deviation $\sigma$ yields insight into the statistical variation about the distant-dependent mean path loss [61].

The CI model ties path loss at any frequency to the physical free space path loss at $1 \mathrm{~m}$ according to Friis' free space equation [85], and has been shown to be robust and accurate in various scenarios [19], [24], [27], [28]. Indoor environments, however, were found to have frequency-dependent loss beyond the first meter, due to the surrounding environment, and work in [21] extended the CI model to the CIF model where the PLE has a frequency-dependent term. Recent work [19], [24] has made $73 \mathrm{GHz}$ rural measurements to beyond $10 \mathrm{~km}$ and adapted the CIF model form to predict path loss as a function of TX antenna height in RMa scenarios, as path loss was found to be accurately predicted with a height dependency in the PLE, leading to the CIH mode ${ }^{1}$, which has the same form of the CIF model given in (4):

\footnotetext{
${ }^{1}$ The CIH model has the same form as (4) except the PLE is a function of the BS height in the RMa scenario instead of frequency, as given by: $P L^{C I H}\left(f_{c}, d, h_{B S}\right) \quad[\mathrm{dB}]=32.4+20 \log _{10}\left(f_{c}\right)+$ $10 n\left(1+b_{t x}\left(\frac{h_{B S}-h_{B 0}}{h_{B 0}}\right)\right) \log _{10}(d)+\chi_{\sigma}$, where $d \geq 1 \mathrm{~m}$, and $h_{B 0}$ is a reference RMa BS height [19].
} 


$$
\begin{aligned}
P L^{C I F}\left(f_{c}, d\right)[\mathrm{dB}] & =32.4+20 \log _{10}\left(f_{c}\right) \\
+ & 10 n\left(1+b\left(\frac{f_{c}-f_{0}}{f_{0}}\right)\right) \log _{10}(d)+\chi_{\sigma}^{C I F}
\end{aligned}
$$

where $n$ denotes the distance dependence of path loss, $b$ is an optimization parameter that describes the linear dependence of path loss about the weighted average of frequencies $f_{0}$ (in $\mathrm{GHz}$ ), from the data used to optimize the model [19], [21], [24].

The CIF model uses two parameters to model average path loss over distance, and reverts to the single parameter CI model when $b=0$ for multiple frequencies, or when a single frequency $f=f_{0}$ is modeled [12], [13], [16], [19], [21].

The FI/ABG path loss model is given as:

$$
P L^{A B G}\left(f_{c}, d\right)[\mathrm{dB}]=10 \alpha \log _{10}(d)+\beta+10 \gamma \log _{10}\left(f_{c}\right)+\chi_{\sigma}^{A B G}
$$

where three model parameters $\alpha, \beta$ and $\gamma$ are determined by finding the best fit values to minimize the error between the model and the measured data. In (5), $\alpha$ indicates the slope of path loss with $\log$ distance, $\beta$ is the floating offset value in $\mathrm{dB}$, and $\gamma$ models the frequency dependence of path loss, where $f_{c}$ is in $\mathrm{GHz}$.

Generalizations of the CI, CIF, and FI/ABG models consider different slopes of path loss over distance before and after a breakpoint distance, where the location of the breakpoint depends mostly on the environment. The dual-slope CIF model is:

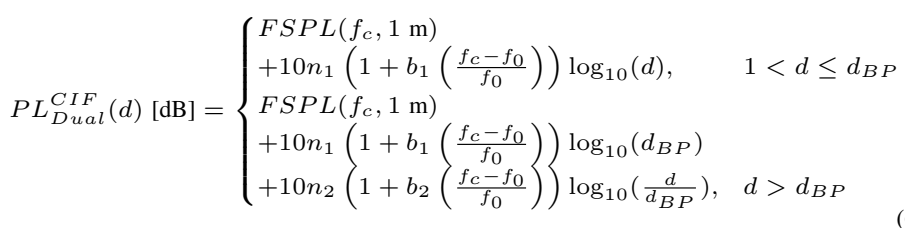

The dual-slope ABG model is:

$$
P L_{\text {Dual }}^{A B G}(d)[\mathrm{dB}]= \begin{cases}\alpha_{1} * 10 \log _{10}(d)+\beta_{1} & \\ +\gamma * 10 \log _{10}\left(f_{c}\right), & 1<d \leq d_{B P} \\ \alpha_{1} * 10 \log _{10}\left(d_{B P}\right)+\beta_{1} & \\ +\gamma * 10 \log _{10}\left(f_{c}\right) & \\ +\alpha_{2} * 10 \log _{10}\left(\frac{d}{d_{B P}}\right), & d>d_{B P}\end{cases}
$$

where the $\alpha_{1}$ and $\alpha_{2}$ are the "dual slope" and $d_{B P}$ is the breakpoint distance. Both dual-slope models require 5 parameters to predict distant-dependent average path loss (frequencies are in $\mathrm{GHz}$ and distances are in meters).

\section{1) UMi Large-Scale Path Loss:}

a) 5GCM: In the 5GCM white paper [12], the CI model (2) is chosen for modeling UMi LOS path loss, since $\alpha$ in the ABG model (5) is almost identical to the PLE of the CI model, and also $\gamma$ is very close to 2 which is predicted by the physically-based Friis' free space equation and used in the CI model [28]. Both the $\mathrm{CI}$ and $\mathrm{ABG}$ models were adopted for UMi NLOS in 5GCM, and the parameters values for the $\mathrm{CI}$ and $\mathrm{ABG}$ models are given in Table IV. In the CI path loss model, only a single parameter, the PLE, needs to be determined through optimization to minimize the model error of mean loss over distance, however, in the ABG model, three parameters need to be optimized to minimize the error, but with very little reduction of the shadowing variance compared to the CI model [21], [28], [83].

b) 3GPP TR 38.901: Path loss models in [101] use 3D T$\mathrm{R}$ separation distances $d_{3 D}$ that account for the BS height $\left(h_{B S}\right)$ and UE height $\left(h_{U E}\right)$. The distribution of the shadow fading is log-normal, and the standard deviation for LOS is $\sigma_{S F}=4.0 \mathrm{~dB}$. The UMi path loss model for LOS is a breakpoint model. For $d_{2 D}<d_{B P}^{\prime}$, the model is essentially a CI model with $n=2.1$

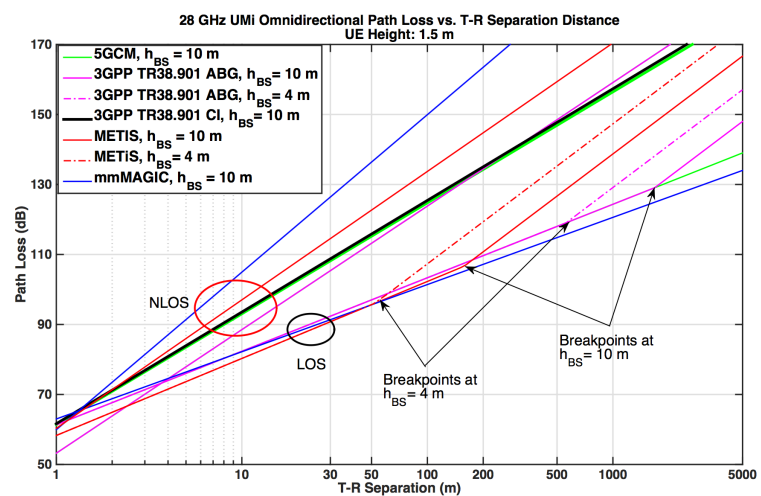

Fig. 5: $P L$ vs. T-R distance comparison among four different path loss models in UMi scenario.

[20], [28], [83], [84], [117]. The LOS breakpoint distance $d_{B P}^{\prime}$ is a function of the carrier frequency, BS height, and the UE height [16], [101]:

$$
\begin{array}{r}
d_{B P}^{\prime}=4 h_{B S}^{\prime} h_{U E}^{\prime} f_{c} \times 10^{9} / c \\
h_{B S}^{\prime}=h_{B S}-1.0 \mathrm{~m}, \\
\\
h_{U E}^{\prime}=h_{U E}-1.0 \mathrm{~m}
\end{array}
$$

where $h_{B S}^{\prime}$ and $h_{U E}^{\prime}$ are the effective antenna heights at the BS and the UE, and $h_{B S}$ and $h_{U E}$ are the actual antenna heights, respectively. The breakpoint distance in an urban environment $[118]$ is where the PLE transitions from free space $(n=2)$ to the asymptotic two-ray ground bounce model of $n=4$ [19], [119]. At mmWave frequencies, the use of a breakpoint is controversial as it has not been reported in measurement, but some ray tracing simulations predict that it will occur [105]. Since the UMi cells radius is typically $500 \mathrm{~m}$ or less, the use of a breakpoint and the height factors in (8) are not necessary (the breakpoint distance is larger than $500 \mathrm{~m}$ even with the smallest possible breakpoint distance when $h_{B S}=4 \mathrm{~m}$ and $h_{U E}=1.5 \mathrm{~m}$ as shown in Fig. 5]. The CI model provides a similar prediction of the path loss with a much simpler equation [2] [84].

In the NLOS scenarios, the UMi-NLOS model uses the ABG model form [114], with a frequency-dependent term that indicates path loss increases with frequency and also has an additional height correction term for the UE. Furthermore, a mathematical patch to correct model deficiencies is used to set a lower bound for the NLOS model as the LOS path loss. The shadow fading standard deviation for UMi NLOS is $\sigma_{S F}=7.82 \mathrm{~dB}$ [16], [22], [108]. The physically-based CI model is also provided as an optional NLOS path loss model for 3GPP TR 38.901 with parameter values given in Table IV

c) METIS: The path loss model for UMi in METIS [102] is a modified version of the ITU-R UMi path loss model [106] and is claimed to be valid for frequencies from 0.8 to $60 \mathrm{GHz}$ (see Table IV). Some METIS models include breakpoints based on sub-6 GHz work (see Fig. 5), yet mmWave measurements to date do not show breakpoints to exist [19], [102], [105]. For LOS scenarios, a scaling factor is used, so that the breakpoint distance $d_{B P}$ (in meters) becomes:

$$
d_{B P}=0.87 \exp \left(-\frac{\log _{10}\left(f_{c}\right)}{0.65}\right) \frac{4\left(h_{B S}-1 \mathrm{~m}\right)\left(h_{U E}-1 \mathrm{~m}\right)}{\lambda}
$$

and the path loss formula for LOS is written as:

$$
P L_{L O S}\left(d_{1}\right)[\mathrm{dB}]=10 n_{1} \log _{10}\left(d_{1}\right)+28.0+20 \log _{10}\left(f_{c}\right)+P L_{0}
$$


TABLE V: Omnidirectional Path loss models in the UMa scenario

\begin{tabular}{|c|c|c|c|}
\hline & PL $[\mathbf{d B}], f_{c}$ is in $\mathbf{G H z}, d$ is in meters & $\begin{array}{c}\text { Shadow fading } \\
\text { std [dB] }\end{array}$ & $\begin{array}{l}\text { Applicability range } \\
\text { and Parameters }\end{array}$ \\
\hline \multicolumn{4}{|c|}{ 5GCM $[\overline{12}]$} \\
\hline $\begin{array}{l}\text { 5GCM UMa } \\
\text { LOS }\end{array}$ & $\begin{array}{c}\text { CI model with } 1 \text { m reference distance: } \\
P L=32.4+20 \log _{10}\left(d_{3 D}\right)+20 \log _{10}\left(f_{c}\right)\end{array}$ & $\sigma_{S F}=4.1$ & $6<f_{c}<100 \mathrm{GHz}$ \\
\hline $\begin{array}{l}\text { 5GCM UMa } \\
\text { NLOS }\end{array}$ & $\begin{array}{c}\text { CI model with 1 m reference distance: } \\
P L=32.4+30 \log _{10}\left(d_{3 D}\right)+20 \log _{10}\left(f_{c}\right) \\
\quad \text { ABG model: } \\
P L=34 \log _{10}\left(d_{3 D}\right)+19.2+23 \log _{10}\left(f_{c}\right) \\
\end{array}$ & $\begin{array}{c}\sigma_{S F}=6.8 \\
\sigma_{S F}=6.5\end{array}$ & $6<f_{c}<100 \mathrm{GHz}$ \\
\hline \multicolumn{4}{|c|}{ 3GPP TR 38.901 V14.0.0 [101] } \\
\hline $\begin{array}{l}\text { 3GPP TR } 38.901 \mathrm{UMa} \\
\text { LOS }\end{array}$ & $\begin{array}{c}P L_{U M a-L O S}= \begin{cases}P L_{1}, & 10 \mathrm{~m} \leq d_{2 D} \leq d_{B P}^{\prime} \\
P L_{2}, & d_{B P}^{\prime} \leq d_{2 D} \leq 5 \mathrm{~km}\end{cases} \\
P L_{1}=28.0+22 \log _{10}\left(d_{3 D}\right)+20 \log _{10}\left(f_{c}\right) \\
P L_{2}=28.0+40 \log _{10}\left(d_{3 D}\right)+20 \log _{10}\left(f_{c}\right) \\
-9 \log _{10}\left(\left(d_{B P}^{\prime}\right)^{2}+\left(h_{B S}-h_{U E}\right)^{2}\right) \\
\text { where } d_{B P}^{\prime}=4 h_{B S}^{\prime} h_{U E}^{\prime} f_{c} \times 10^{9} / c\end{array}$ & $\sigma_{S F}=4.0$ & $\begin{array}{c}0.5<f_{c}<100 \mathrm{GHz} \\
1.5 \mathrm{~m} \leq h_{U E} \leq 22.5 \mathrm{~m} \\
h_{B S}=25 \mathrm{~m}\end{array}$ \\
\hline $\begin{array}{l}\text { 3GPP TR } 38.901 \mathrm{UMa} \\
\text { NLOS }\end{array}$ & $\begin{array}{c}P L=\max \left(P L_{U M a-L O S}\left(d_{3 D}\right), P L_{U M a-N L O S}\left(d_{3 D}\right)\right) \\
P L_{U M a-N L O S}=13.54+39.08 \log _{10}\left(d_{3 D}\right)+20 \log _{10}\left(f_{c}\right) \\
-0.6\left(h_{U E}-1.5\right) \\
\text { Option: CI model with } \mathbf{1} \text { m reference distance } \\
P L=32.4+20 \log _{10}\left(f_{c}\right)+30 \log _{10}\left(d_{3 D}\right)\end{array}$ & $\begin{array}{l}\sigma_{S F}=6.0 \\
\sigma_{S F}=7.8\end{array}$ & $\begin{array}{c}0.5<f_{c}<100 \mathrm{GHz} \\
10 \mathrm{~m}<d_{2 D}<5000 \mathrm{~m} \\
1.5 \mathrm{~m} \leq h_{U E} \leq 22.5 \mathrm{~m} \\
h_{B S}=25 \mathrm{~m}\end{array}$ \\
\hline \multicolumn{4}{|c|}{ METIS $[102]$} \\
\hline $\begin{array}{l}\text { METIS UMa } \\
\text { LOS }\end{array}$ & $\begin{array}{c}P L_{U M a-L O S}= \begin{cases}P L_{1}, & 10 \mathrm{~m} \leq \overline{d_{2 D}} \leq d_{B P}^{\prime} \\
P L_{2}, & d_{B P}^{\prime} \leq d_{2 D} \leq 5 \mathrm{~km}\end{cases} \\
P L_{1}=28+22 \log _{10}\left(d_{3 D}\right)+20 \log _{10}\left(f_{c}\right) \\
P L_{2}=28+40 \log _{10}\left(d_{3 D}\right)+20 \log _{10}\left(f_{c}\right) \\
-9 \log _{10}\left(\left(d_{B P}^{\prime}\right)^{2}+\left(h_{B S}-h_{U E}\right)^{2}\right) \\
\text { where } d_{B P}^{\prime}=4\left(h_{B S}-1\right)\left(h_{U E}-1\right) f_{c} \times 10^{9} / c\end{array}$ & $\sigma_{S F}=4.0$ & $\begin{array}{c}0.45<f_{c}<6 \mathrm{GHz} \\
10 \mathrm{~m}<d_{2 D}<5000 \mathrm{~m} \\
1.5 \mathrm{~m} \leq h_{U E} \leq 22.5 \mathrm{~m} \\
h_{B S}=25 \mathrm{~m}\end{array}$ \\
\hline $\begin{array}{l}\text { METIS UMa } \\
\text { NLOS }\end{array}$ & $\begin{array}{c}P L=\max \left(P L_{U M a-L O S}\left(d_{3 D}\right), P L_{U M a-N L O S}\left(d_{3 D}\right)\right) \\
P L_{U M a-N L O S}=161.94-7.1 \log _{10}(w)+7.5 \log _{10}(h) \\
-\left(24.37-3.7\left(\frac{h}{h_{B S}}\right)^{2}\right) \log _{10}\left(h_{B S}\right) \\
+\left(43.42-3.1 \log _{10}\left(h_{B S}\right)\right)\left(\log _{10}\left(d_{3 D}\right)-3\right) \\
+20 \log _{10}\left(f_{c}\right)-0.6\left(h_{U E}\right) \\
\end{array}$ & $\sigma_{S F}=6.0$ & $\begin{array}{c}0.45<f_{c}<6 \mathrm{GHz} \\
10 \mathrm{~m}<d_{2 D}<5000 \mathrm{~m} \\
1.5 \mathrm{~m} \leq h_{U E} \leq 22.5 \mathrm{~m} \\
h_{B S}=25 \mathrm{~m} \\
w=20 \mathrm{~m} \\
h=20 \mathrm{~m}\end{array}$ \\
\hline
\end{tabular}

for $10 \mathrm{~m}<d \leqslant d_{B P}$, where $P L_{0}$ is a path loss offset calculated by:

$$
P L_{0}[\mathrm{~dB}]=-1.38 \log _{10}\left(f_{c}\right)+3.34
$$

Path loss after the breakpoint distance is:

$$
P L_{L O S}\left(d_{1}\right)[\mathrm{dB}]=10 n_{2} \log _{10}\left(\frac{d_{1}}{d_{B P}}\right)+P L_{L O S}\left(d_{B P}\right)
$$

for $d_{B P}<d_{1}<500 \mathrm{~m}$ where (10) and (12) represent path loss before and after the breakpoint, respectively. The last term $P L\left(d_{B P}\right)$ in 12 is derived from 10 by substituting $d_{1}$ with $d_{B P}$ to calculate path loss at the breakpoint distance [102].

The UMi NLOS path loss model in METIS is adopted from the 3GPP TR 36.873 [96], [102] sub-6 GHz model for 4G LTE and is calculated as:

$$
\begin{aligned}
& P L=\max \left(P L_{L O S}\left(d_{3 D}\right), P L_{N L O S}\left(d_{3 D}\right)\right) \\
& P L_{N L O S}=36.7 \log _{10}\left(d_{3 D}\right)+23.15+26 \log _{10}\left(f_{c}\right)-0.3\left(h_{U E}\right)
\end{aligned}
$$

where $f_{c}$ is in $\mathrm{GHz}, 10 \mathrm{~m}<d_{3 D}<2000 \mathrm{~m}$, and $1.5 \mathrm{~m} \leq h_{U E} \leq$ $22.5 \mathrm{~m}$.

d) mmMAGIC: The mmMAGIC project [92] adopted the ABG path loss model for UMi, similar to that from 5GCM [12] but with different parameter values (see Table IV). Comparisons among the different UMi large-scale path loss models described here are provided in Fig. 5 .

2) UMa Large-Scale Path Loss:

a) 3GPP TR 38.901: The 3GPP TR 38.901 [101] UMa LOS path loss model is adopted from 3GPP TR 36.873 (below $6 \mathrm{GHz}$ Release 12 for LTE) [96] and TR 38.900 [112], [120]. For the
UMa NLOS scenario, an ABG model and an optional CI model are provided (see Table $\mathrm{V}$ for parameters). With respect to the UMa LOS model, 3GPP TR 38.901 inexplicably discards the TR 38.900 [112] model and reverts back to TR 36.873 which is defined only for below $6 \mathrm{GHz}$ [96] while also omitting the $\mathrm{InH}$ shopping mall scenario used in TR 38.900. TR 38.901 models omnidirectional path loss from $0.5-100 \mathrm{GHz}$, but lacks measurement validation in some cases.

b) 5GCM: There are three UMa path loss models used in [12]: CI, CIF, and ABG [28], [84]. The PLEs of the CI/CIF models for UMa are somewhat lower than for the UMi models indicating less loss over distance, which makes sense intuitively since a larger BS height implies that fewer obstructions are encountered than in the UMi scenario [27].

c) METIS: METIS adopted the sub-6 GHz 3GPP TR 36.873 [96] 3D UMa model that was published in 2014 for LTE, see Table V]

\section{3) InH Large-Scale Path Loss:}

a) 5GCM: In the InH scenario, besides the CI, CIF, and ABG path loss models, dual-slope path loss models are proposed for different distance zones in the propagation environment and are provided in Table VI. For NLOS, both the dual-slope ABG and dual-slope CIF models are considered for $5 \mathrm{G}$ performance evaluation, where they each require five modeling parameters to be optimized. Also, a single-slope CIF model that uses only two optimization parameters is considered for InH-Office [12], [21]. The dual-slope model may be best suited for InH-shopping mall or large indoor distances (greater than $50 \mathrm{~m}$ ), although it is not clear 
TABLE VI: 5GCM omnidirectional path loss models in the InH scenario

\begin{tabular}{|c|c|c|c|}
\hline & PL $[\mathbf{d B}], f_{c}$ is in $\mathbf{G H z}, d$ is in meters & $\begin{array}{c}\text { Shadow fading } \\
\text { std [dB] }\end{array}$ & $\begin{array}{l}\text { Applicability range } \\
\text { and Parameters }\end{array}$ \\
\hline $\begin{array}{l}\text { 5GCM InH } \\
\text { Indoor-Office } \\
\text { LOS }\end{array}$ & $\begin{array}{c}\text { CI model with } 1 \text { m reference distance: } \\
P L=32.4+17.3 \log _{10}\left(d_{3 D}\right)+20 \log _{10}\left(f_{c}\right)\end{array}$ & $\sigma_{S F}=3.02$ & $6<f_{c}<100 \mathrm{GHz}$ \\
\hline $\begin{array}{l}\text { 5GCM InH } \\
\text { Indoor-Office } \\
\quad \text { NLOS } \\
\text { single slope (FFS) }\end{array}$ & $\begin{array}{c}\text { CIF model: } \\
P L=32.4+31.9\left(1+0.06\left(\frac{f_{c}-24.2}{24.2}\right)\right) \log _{10}\left(d_{3 D}\right)+20 \log _{10}\left(f_{c}\right) \\
\text { ABG model: } \\
P L=38.3 \log _{10}\left(d_{3 D}\right)+17.30+24.9 \log _{10}\left(f_{c}\right)\end{array}$ & $\begin{array}{l}\sigma_{S F}^{C I F}=8.29 \\
\sigma_{S F}^{A B G}=8.03\end{array}$ & $6<f_{c}<100 \mathrm{GHz}$ \\
\hline $\begin{array}{l}\text { 5GCM InH } \\
\text { Indoor-Office } \\
\text { NLOS } \\
\text { dual slope }\end{array}$ & 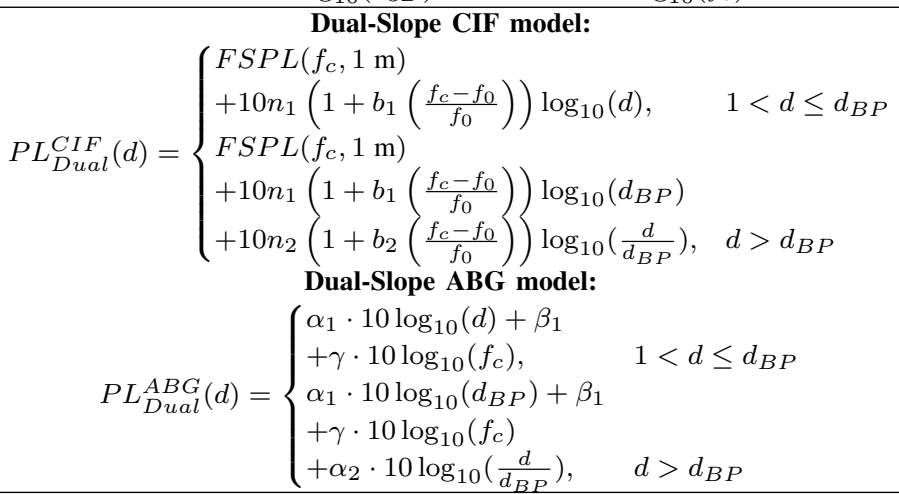 & $\begin{aligned} \sigma_{S F}^{C I F} & =7.65 \\
\sigma_{S F}^{A B G} & =7.78\end{aligned}$ & $\begin{array}{c}6<f_{c}<100 \mathrm{GHz} \\
\text { Dual-Slope CIF model: } \\
n_{1}=2.51, b=0.06 \\
f_{0}=24.1 \mathrm{GHz}, n_{2}=4.25 \\
b_{2}=0.04, d_{B P}=7.8 \mathrm{~m} \\
\text { Dual-Slope ABG model: } \\
\alpha_{1}=1.7, \beta_{1}=33.0 \\
\gamma=2.49, d_{B P}=6.9 \mathrm{~m} \\
\alpha_{2}=4.17\end{array}$ \\
\hline $\begin{array}{l}\text { 5GCM InH } \\
\text { Shopping-Mall } \\
\text { LOS }\end{array}$ & $\begin{array}{c}\text { CI model with } 1 \text { m reference distance: } \\
P L=32.4+17.3 \log _{10}\left(d_{3 D}\right)+20 \log _{10}\left(f_{c}\right)\end{array}$ & $\sigma_{S F}=2.01$ & $6<f_{c}<100 \mathrm{GHz}$ \\
\hline $\begin{array}{l}\text { 5GCM InH } \\
\text { Shopping-Mall } \\
\text { NLOS } \\
\text { single slope (FFS) }\end{array}$ & $\begin{array}{c}\text { CIF model: } \\
P L=32.4+25.9\left(1+0.01\left(\frac{f_{c}-39.5}{39.5}\right)\right) \log _{10}\left(d_{3 D}\right)+20 \log _{10}\left(f_{c}\right) \\
\text { ABG model: } \\
P L=32.1 \log _{10}\left(d_{3 D}\right)+18.09+22.4 \log _{10}\left(f_{c}\right)\end{array}$ & $\begin{array}{l}\sigma_{S F}^{C I F}=7.40 \\
\sigma_{S F}^{A B G}=6.97\end{array}$ & $6<f_{c}<100 \mathrm{GHz}$ \\
\hline $\begin{array}{l}\text { 5GCM InH } \\
\text { Shopping-Mall } \\
\text { NLOS } \\
\text { dual slope }\end{array}$ & 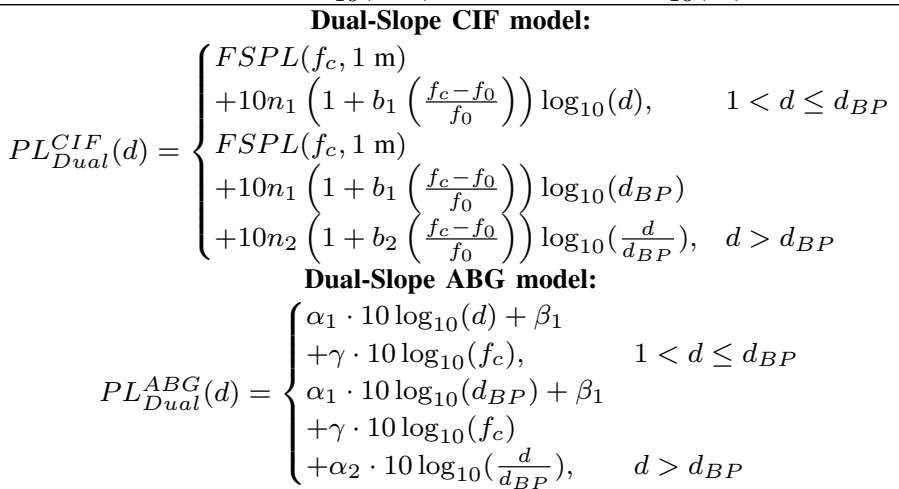 & $\begin{aligned} \sigma_{S F}^{C I F} & =6.26 \\
\sigma_{S F}^{A B G} & =6.36\end{aligned}$ & $\begin{array}{c}6<f_{c}<100 \mathrm{GHz} \\
\text { Dual-Slope CIF model: } \\
n_{1}=2.43, b=-0.01 \\
f_{0}=39.5 \mathrm{GHz}, n_{2}=8.36 \\
b_{2}=0.39, d_{B P}=110 \mathrm{~m} \\
\text { Dual-Slope ABG model: } \\
\alpha_{1}=2.9, \beta_{1}=22.17 \\
\gamma=2.24, d_{B P}=147.0 \mathrm{~m} \\
\alpha_{2}=11.47\end{array}$ \\
\hline
\end{tabular}

from the data in [12] that the additional complexity is warranted when compared to the simple CIF model.

b) 3GPP TR 38.901: The path loss model for the InH-office LOS scenario in 3GPP TR 38.901 [101] is claimed to be valid up to $100 \mathrm{~m}$ and has the same form as the CI model in the UMi scenario. The only differences from UMi CI model are that the PLE in InH-office is slightly lower than that in the UMi street canyon due to more reflections and scattering in the indoor environment from walls and ceilings and waveguiding effects down hallways that increase received signal power [21].

The 3GPP TR 38.901 InH-office NLOS path loss model uses the ABG model form similar to its UMi NLOS path loss model, except that there is no height correction term, and the model requires a patch to ensure it is lower-bounded by the LOS path loss as follows:

$$
P L[\mathrm{~dB}]=\max \left(P L_{I n H-L O S}\left(d_{3 D}\right), P L_{I n H-N L O S}\left(d_{3 D}\right)\right)
$$

$$
P L_{I n H-N L O S}[\mathrm{~dB}]=17.30+38.3 \log _{10}\left(d_{3 D}\right)+24.9 \log _{10}\left(f_{c}\right)
$$

c) METIS: In the latest METIS white paper [102], the WINNER II path loss model (similar in form to the ABG model) was adopted as the geometry-based stochastic model for short-range 60 $\mathrm{GHz}(61-65 \mathrm{GHz})$ links in indoor environments:

$$
P L[\mathrm{~dB}]=A \log _{10}(d)+B
$$

where $A$ and $B$ are curve-fit parameters without the use of Friis' equation [85] (see Table VII for parameters).

d) mmMAGIC: The InH channel model in mmMAGIC [92] is adopted from an earlier version of 5GCM [12], and has the same form as the ABG model. For Indoor-NLOS, the values of the path loss model parameters have been averaged from $\mathrm{InH}$ and InH-Shopping Mall.

e) IEEE 802.11ad: In the STA-STA (STA signifies a station, the WiFi term for the UE) LOS scenario [69], path loss follows theoretical free space path loss in the CI model form via the Friis' free space transmission equation as given in Table VII No shadowing term is provided in the LOS case, as instantaneous realizations are claimed to be close to the average path loss value over such wideband channel bandwidth.

Experiments performed for NLOS situations resulted in path loss for STA-STA as a FI/AB model [20] with the shadow fading standard deviation as $\sigma_{S F}=3.3 \mathrm{~dB}$. The $2 \mathrm{D}$ distance $d_{2 D}$ is used 
TABLE VII: Other omnidirectional path loss models in the InH scenario

\begin{tabular}{|c|c|c|c|}
\hline & PL $[\mathbf{d B}], f_{c}$ is in $\mathbf{G H z}, d$ is in meters & $\begin{array}{l}\text { Shadow fading } \\
\quad \text { std }[\mathrm{dB}]\end{array}$ & $\begin{array}{l}\text { Applicability range } \\
\text { and Parameters }\end{array}$ \\
\hline \multicolumn{4}{|c|}{ 3GPP TR 38.901 V14.0.0 |101] } \\
\hline $\begin{array}{l}\text { 3GPP TR } 38.901 \\
\text { Indoor-Office LOS }\end{array}$ & $P L_{I n H-L O S}=32.4+17.3 \log _{10}\left(d_{3 D}\right)+20 \log _{10}\left(f_{c}\right)$ & $\sigma_{S F}=3.0$ & $\begin{array}{c}0.5<f_{c}<100 \mathrm{GHz} \\
1<d_{3 D}<100 \mathrm{~m}\end{array}$ \\
\hline $\begin{array}{l}\text { 3GPP TR } 38.901 \\
\text { Indoor-Office NLOS }\end{array}$ & $\begin{array}{c}P L=\max \left(P L_{I n H-L O S}\left(d_{3 D}\right), P L_{I n H-N L O S}\left(d_{3 D}\right)\right) \\
P L_{I n H-N L O S}=17.30+38.3 \log _{10}\left(d_{3 D}\right)+24.9 \log _{10}\left(f_{c}\right) \\
\text { Option: CI model with } 1 \text { m reference distance } \\
P L=32.4+20 \log _{10}\left(f_{c}\right)+31.9 \log _{10}\left(d_{3 D}\right)\end{array}$ & $\begin{array}{c}\sigma_{S F}=8.03 \\
\sigma_{S F}=8.29\end{array}$ & $\begin{aligned} 0.5 & <f_{c}<100 \mathrm{GHz} \\
1 & <d_{3 D}<86 \mathrm{~m} \\
1 & <d_{3 D}<86 \mathrm{~m}\end{aligned}$ \\
\hline \multicolumn{4}{|c|}{ METIS 102 } \\
\hline $\begin{array}{c}\text { METIS } \\
\text { Shopping Mall LOS }\end{array}$ & $P L=68.8+18.4 \log _{10}\left(d_{2 D}\right)$ & $\sigma_{S F}=2.0$ & $\begin{array}{c}f_{c}=63 \mathrm{GHz} \\
1.5<d_{2 D}<13.4 \mathrm{~m} \\
h_{B S}=h_{U E}=2 \mathrm{~m}\end{array}$ \\
\hline $\begin{array}{c}\text { METIS } \\
\text { Shopping Mall NLOS }\end{array}$ & $P L=94.3+3.59 \log _{10}\left(d_{2 D}\right)$ & $\sigma_{S F}=2.0$ & $\begin{array}{c}f_{c}=63 \mathrm{GHz} \\
4<d_{2 D}<16.1 \mathrm{~m} \\
h_{B S}=h_{U E}=2 \mathrm{~m}\end{array}$ \\
\hline \multicolumn{4}{|c|}{ IEEE 802.11ad $|69|$} \\
\hline $\begin{array}{l}\text { 802.11ad } \\
\text { Indoor-Office LOS }\end{array}$ & $P L_{L O S}[d B]=32.5+20 \log _{10}\left(f_{c}\right)+20 \log _{10}\left(d_{2 D}\right)$ & $\sigma_{S F}$ & $57<f_{c}<63 \mathrm{GHz}$ \\
\hline $\begin{array}{c}\text { 802.11ad } \\
\text { Indoor-Office NLOS }\end{array}$ & $\begin{array}{c}P L_{N L O S}[d B]=51.5+20 \log _{10}\left(f_{c}\right)+6 \log _{10}\left(d_{2 D}\right) \\
P L_{N L O S}[d B]=45.5+20 \log _{10}\left(f_{c}\right)+14 \log _{10}\left(d_{3 D}\right)\end{array}$ & $\begin{aligned} \sigma_{S F}^{S T A-S T A} & =3.3 \\
\sigma_{S F}^{S T A-A P} & =3\end{aligned}$ & $57<f_{c}<63 \mathrm{GHz}$ \\
\hline \multicolumn{4}{|c|}{ mmMAGIC |92| } \\
\hline $\begin{array}{l}\text { mmMAGIC InH } \\
\text { LOS }\end{array}$ & $P L_{L O S}=13.8 \log _{10}\left(d_{3 D}\right)+33.6+20.3 \log _{10}\left(f_{c}\right)$ & $\sigma_{S F}=1.18$ & $6<f_{c}<100 \mathrm{GHz}$ \\
\hline $\begin{array}{l}\text { mmMAGIC InH } \\
\text { NLOS }\end{array}$ & $\begin{array}{c}P L=\max \left(P L_{L O S}\left(d_{3 D}\right), P L_{N L O S}\left(d_{3 D}\right)\right) \\
P L_{N L O S}=36.9 \log _{10}\left(d_{3 D}\right)+15.2+26.8 \log _{10}\left(f_{c}\right)\end{array}$ & $\sigma_{S F}=8.03$ & $6<f_{c}<100 \mathrm{GHz}$ \\
\hline
\end{tabular}

for the STA-STA scenario, since it is considered that two stations are deemed to be at the same height above ground.

In the STA-AP (where the AP denotes access point, corresponding to a BS) scenario, the 3D separation distance $d_{3 D}$ is used, and the LOS STA-AP path loss model is the same CI model as used in the STA-STA situation but no specific shadow fading term is given. The NLOS STA-AP model takes the same ABG form as that of STA-STA, but with $A_{N L O S}=45.5 \mathrm{~dB}$ and a shadow fading standard deviation $\sigma_{S F}=3.0 \mathrm{~dB}$.

4) RMa Large-Scale Path Loss:

a) 3GPP TR 38.901: The 3GPP TR 38.901 RMa path loss model [101] is mostly adopted from sub-6 GHz ITU-R M.2135 [106] as described below, and claims validity up to 30 $\mathrm{GHz}$, based on a single $24 \mathrm{GHz}$ measurement campaign over short distances less than $500 \mathrm{~m}$ and without any goodness of fit indication [121]. Work in [19], [24] advocates a much more fundamental and accurate RMa model using the CIF model formulation in (4), where the frequency dependency of the PLE is replaced with a TX height dependency of the PLE, based on many propagation studies that showed UMa and RMa environment did not offer additional frequency dependency of the path loss over distance beyond the first meter of propagation [19], [24], [28], [83].

b) ITU-R: The ITU-R communication sector published guidelines for the evaluation of radio interface technologies for IMTAdvanced in ITU-R M.2135 which is valid for sub-6 GHz [106]. The rural scenario is best described as having BS heights of 35 $\mathrm{m}$ or higher, generally much higher than surrounding buildings. The LOS path loss model has a controversial breakpoint distance
[19] and a maximum 2D T-R separation distance of $10 \mathrm{~km}$, while the NLOS path loss model has a maximum 2D T-R separation distance of $5 \mathrm{~km}$ with no breakpoint distance. Initial antenna height default values are provided in Table VIII] with the following four correction factor parameters: street width $W$, building height $h$, BS height $h_{B S}$, and UE height $h_{U E}$ (all in meters).

The ITU-R RMa LOS path loss model is quite complex:

$$
\begin{aligned}
P L_{1}[\mathrm{~dB}] & =20 \log \left(40 \pi \cdot d_{3 D} \cdot f_{c} / 3\right) \\
& +\min \left(0.03 h^{1.72}, 10\right) \log _{10}\left(d_{3 D}\right) \\
& -\min \left(0.044 h^{1.72}, 14.77\right)+0.002 \log _{10}(h) d_{3 D} \\
P L_{2}[\mathrm{~dB}] & =P L_{1}\left(d_{B P}\right)+40 \log _{10}\left(d_{3 D} / d_{B P}\right)
\end{aligned}
$$

where the breakpoint distance $d_{B P}$ is:

$$
d_{B P}=2 \pi \cdot h_{B S} \cdot h_{U E} \cdot f_{c} / c
$$

It is must be noted that the model reverts to a single-slope model at $9.1 \mathrm{GHz}$ or above, since the breakpoint distance exceeds 10 $\mathrm{km}$ (the outer limit of model applicability), thus making the LOS model mathematically inconsistent for mmWave frequencies above 9.1 GHz [19], [24].

The NLOS RMa path loss model in (19) is adopted from ITUR M.2135 and has nine empirical coefficients for various building height and street width parameters [101], [106]:

$$
\begin{aligned}
& P L[\mathrm{~dB}]=\max \left(P L_{R M a-L O S}, P L_{R M a-N L O S}\right) \\
& P L_{R M a-N L O S}[\mathrm{~dB}]=161.04-7.1 \log _{10}(W)+7.5 \log _{10}(h) \\
& \quad-\left(24.37-3.7\left(h / h_{B S}\right)^{2}\right) \log _{10}\left(h_{B S}\right) \\
& \quad+\left(43.42-3.1 \log _{10}\left(h_{B S}\right)\right)\left(\log _{10}\left(d_{3 D}\right)-3\right) \\
& \quad+20 \log _{10}\left(f_{c}\right)-\left(3.2\left(\log _{10}\left(11.75 h_{U E}\right)\right)^{2}-4.97\right)
\end{aligned}
$$


TABLE VIII: ITU-R M.2135/3GPP RMa path loss model default values and applicability ranges [101], [106].

\begin{tabular}{|l|}
\hline RMa LOS Default Values Applicability Range \\
\hline $10 \mathrm{~m}<d_{2 D}<d_{B P}$, \\
$d_{B P}<d_{2 D}<10000 \mathrm{~m}$, \\
$h_{B S}=35 \mathrm{~m}, h_{U E}=1.5 \mathrm{~m}, W=20 \mathrm{~m}, h=5 \mathrm{~m}$ \\
Applicability ranges: $5 \mathrm{~m}<h<50 \mathrm{~m} ; 5 \mathrm{~m}<W<50 \mathrm{~m} ;$ \\
$10 \mathrm{~m}<h_{B S}<150 \mathrm{~m} ; 1 \mathrm{~m}<h_{U E}<10 \mathrm{~m}$ \\
\hline RMa NLOS Default Values Applicability Range \\
\hline $10 \mathrm{~m}<d_{2 D}<5000 \mathrm{~m}$, \\
$h_{B S}=35 \mathrm{~m}, h_{U E}=1.5 \mathrm{~m}, W=20 \mathrm{~m}, h=5 \mathrm{~m}$ \\
Applicability ranges: $5 \mathrm{~m}<h<50 \mathrm{~m} ; 5 \mathrm{~m}<W<50 \mathrm{~m} ;$ \\
$10 \mathrm{~m}<h_{B S}<150 \mathrm{~m} ; 1 \mathrm{~m}<h_{U E}<10 \mathrm{~m}$ \\
\hline
\end{tabular}

The ITU-R RMa NLOS path loss model from which the 3GPP TR38.901 model is adopted is only specified for frequencies up to $6 \mathrm{GHz}$ and has not been validated in the literature for mmWave frequencies. The ITU-R RMa models were not developed using rural scenarios [19], [24], but instead were derived from measurements in downtown Tokyo, making them ill-suited for the RMa case.

c) NYU RMa model: NYU proposed empirically-based CIH RMa path loss models for LOS $\left(P L_{L O S}^{C I H-R M a}\right)$ and NLOS $\left(P L_{N L O S}^{C I H-R M a}\right)$ from extensive simulations and $73 \mathrm{GHz}$ field data [19]:

$$
\begin{aligned}
& P L_{L O S}^{C I H-R M a}\left(f_{c}, d, h_{B S}\right)[\mathrm{dB}]=32.4+20 \log _{10}\left(f_{c}\right) \\
& \quad+23.1\left(1-0.03\left(\frac{h_{B S}-35}{35}\right)\right) \log _{10}(d)+\chi_{\sigma_{L O S}}
\end{aligned}
$$

where $d \geq 1 \mathrm{~m}, \sigma_{L O S}=1.7 \mathrm{~dB}$, and $10 \mathrm{~m} \leq h_{B S} \leq 150 \mathrm{~m}$.

$$
\begin{aligned}
& P L_{N L O S}^{C I H-R M a}\left(f_{c}, d, h_{B S}\right)[\mathrm{dB}]=32.4+20 \log _{10}\left(f_{c}\right) \\
& \quad+30.7\left(1-0.049\left(\frac{h_{B S}-35}{35}\right)\right) \log _{10}(d)+\chi_{\sigma_{N L O S}}
\end{aligned}
$$

where $d \geq 1 \mathrm{~m}, \sigma_{L O S}=6.7 \mathrm{~dB}$, and $10 \mathrm{~m} \leq h_{B S} \leq 150 \mathrm{~m}$.

\section{O2I Penetration Loss}

1) 3GPP TR 38.901: The overall large-scale path loss models may also account for penetration loss into a building and subsequent path loss inside the building. The O2I path loss model taking account of the building penetration loss according to 3GPP TR 38.901 [101] has the following form:

$$
P L[\mathrm{~dB}]=P L_{b}+P L_{t w}+P L_{i n}+N\left(0, \sigma_{P}^{2}\right)
$$

where $P L_{b}$ is the basic outdoor path loss, $P L_{t w}$ is the building penetration loss through the external wall, $P L_{i n}$ is the indoor loss which depends on the depth into the building, and $\sigma_{P}$ is the standard deviation for the penetration loss. The building penetration loss $P L_{t w}$ can be modeled as:

$$
P L_{t w}[\mathrm{~dB}]=P L_{n p i}-10 \log _{10} \sum_{i=1}^{N}\left(p_{i} \times 10^{\frac{L_{\text {material }_{i}}}{-10}}\right)
$$

where $P L_{n p i}$ is an additional loss which is added to the external wall loss to account for non-perpendicular incidence, $L_{\text {material }_{i}}=$ $a_{\text {material }_{i}}+b_{\text {material }_{i}} \cdot f_{c}$ is the penetration loss of material $i, f_{c}$ is the frequency in $\mathrm{GHz}, p_{i}$ is the proportion of $i$-th materials, where $\sum p_{i}=1$, and $N$ is the number of materials. Penetration loss of several materials and the O2I penetration loss models are given in Table IX

Rough models are also provided to estimate the building penetration loss in Table $\mathrm{X}$ Both the low-loss and high-loss models are
TABLE IX: O2I penetration loss of different materials [101]

\begin{tabular}{|c|c|}
\hline Material & Penetration loss $[\mathbf{d B}], f_{c}$ is in $\mathbf{G H z}$ \\
\hline Standard multi-pane glass & $L_{\text {glass }}=2+0.2 \cdot f_{c}$ \\
\hline IRR glass & $L_{\text {IRRglass }}=23+0.3 \cdot f_{c}$ \\
\hline Concrete & $L_{\text {concrete }}=5+4 \cdot f_{c}$ \\
\hline Wood & $L_{\text {wood }}=4.85+0.12 \cdot f_{c}$ \\
\hline
\end{tabular}

applicable to UMa and UMi-street canyon, while only the low-loss model is applicable to RMa. The O2I car penetration loss included in path loss is determined by:

$$
P L[\mathrm{~dB}]=P L_{b}+N\left(\mu, \sigma_{P}^{2}\right)
$$

where $P L_{b}$ is the basic outdoor path loss, and for most cases, $\mu=9 \mathrm{~dB}$ and $\sigma_{P}=5 \mathrm{~dB}$. An optional $\mu=20 \mathrm{~dB}$ is provided for metalized car windows for frequencies ranging from 0.6 to 60 $\mathrm{GHz}$ [101].

2) 5GCM: The 5GCM adopted the building penetration loss model of 3GPP TR 36.873 which is based on legacy measurements below $6 \mathrm{GHz}$ [96]. Several different frequency-dependent models were also proposed in [12], [16]. In [109], a detailed description of external wall penetration loss using a composite approach is provided. The difference of the building penetration loss model between 5GCM and 3GPP TR 38.901 is that the standard deviation is tentatively selected from the measurement data [16], [109]. A very simple parabolic model with a good fit for predicting building penetration loss (BPL) of either high loss or low loss buildings was provided in [16], [99] as:

$$
B P L[\mathrm{~dB}]=10 \log _{10}\left(A+B \cdot f_{c}^{2}\right)
$$

where $f_{c}$ is in $\mathrm{GHz}, A=5$, and $B=0.03$ for low loss buildings and $A=10$ and $B=5$ for high loss buildings.

3) mmMAGIC: The O2I penetration loss model in mmMAGIC has the form of:

$$
O 2 I[\mathrm{~dB}]=B_{O 2 I}+C_{O 2 I} \cdot \log _{10}\left(f_{c}\right) \approx 8.5+11.2 \cdot \log _{10}\left(f_{c}\right)
$$

The advantage of this form is that the coefficients $B_{O 2 I}$ and $C_{O 2 I}$ can be added to the existing coefficients in the path loss model of mmMAGIC. A frequency-dependent shadow fading between 8 and $10 \mathrm{~dB}$ for the UMi-O2I scenario is presented in [92]:

$$
\Sigma_{S F}[\mathrm{~dB}]=\sigma_{S F}+\delta_{S F} \cdot \log _{10}\left(f_{c}\right) \approx 5.7+2.3 \cdot \log _{10}\left(f_{c}\right)
$$

\section{Spatial consistency}

Many previous channel models were "drop-based", where a UE is placed at a random location, random channel parameters (conditioned on this location) are assigned, performance is computed (possibly when moving over a short distance, up to 40 wavelengths), and then a different location is chosen at random. This approach is useful for statistical or monte-carlo performance analysis, but does not provide spatial consistency, i.e., two UEs that are dropped at nearly identical T-R separation distances might experience completely different channels from a system simulator. The importance of spatial consistency is dependent upon the sitespecific propagation in a particular location as shown in [75], [91]. Channel models of 5GCM [12], 3GPP TR 38.901 [101], METIS [102] and MiWEBA [104] provide new approaches for modeling of trajectories to retain spatial consistency.

In 5GCM and 3GPP, both the LOS/NLOS state and the shadowing states are generated on a coarse grid, and spatially filtered. This resulting "map" of LOS states and shadowing attenuations are then 
TABLE X: O2I penetration loss parameters [12], [101]

\begin{tabular}{|c|c|c|c|}
\hline & $\begin{array}{c}\text { Path loss through external wall: } \\
P L_{t w}[\mathbf{d B}], f_{c} \text { is in } \mathbf{G H z}\end{array}$ & $\begin{array}{c}\text { Indoor loss: } \\
P L_{i n}[\mathbf{d B}], \mathbf{d} \text { is in meters }\end{array}$ & $\begin{array}{l}\text { Standard deviation: } \\
\qquad \sigma_{P}[\mathrm{~dB}]\end{array}$ \\
\hline 3GPP TR 38.901 Low-loss model [101] & $5-10 \log _{10}\left(0.3 \cdot 10^{-L_{\text {glass }} / 10}+0.7 \cdot 10^{-L_{\text {concrete }} / 10}\right)$ & $0.5 d_{2 D-i n}$ & 4.4 \\
\hline 3GPP TR 38.901 High-loss model [101] & $5-10 \log _{10}\left(0.7 \cdot 10^{-L_{\text {IRRglass }} / 10}+0.3 \cdot 10^{-L_{\text {concrete }} / 10}\right)$ & $0.5 d_{2 D-i n}$ & 6.5 \\
\hline 5GCM Low-loss model [12], [99] & $10 \log _{10}\left(5+0.03 \cdot f_{c}^{2}\right)$ & Not Specified & 4.0 \\
\hline 5GCM High-loss model $|\overline{12}|,|\overline{99}|$ & $10 \log _{10}\left(10+5 \cdot f_{c}^{2}\right)$ & Not Specified & 6.0 \\
\hline
\end{tabular}

used for the trajectories of all UEs during the simulation process. For the implementation of the LOS state filtering, different methods are proposed [12], [101], but the effect is essentially the same. We note that 5GCM and $3 \mathrm{GPP}$ also introduce additional procedures to ensure spatial consistencies of the delay and angles, but those considerations are beyond the scope of this paper. The map-based models of METIS [102] and MiWEBA [104] inherently provide spatial consistency, as the dominant paths for close-by locations are identical, and their effect is computed deterministically. Generally speaking, spatial consistency is easier to implement in geometrybased models (such as semi-deterministic and geometric-based stochastic channel models) than in tapped-delay line models such as 3GPP. Work in [12], [29], [75], [91], [92] shows that the degree of spatial consistency can vary widely at mmWave frequencies.

\section{CONCLUSION}

Often times, standard bodies have additional reasons to adopt particular modeling formulations, beyond physical laws or the fitting of data to observed channel characteristics. Motivations often include ensuring simulations work for legacy software at lower frequencies, or the desire to rapidly converge while preserving legacy approaches (see [19], [28], [80], [111] for example). Channel modeling for $5 \mathrm{G}$ is an on-going process and early results show significant capacity differences arise from different models [80], [99], [100]. Futher work is needed to bolster and validate the early channel models. Many new mmWave channel simulators (e.g., NYUSIM, QuaDRiGa) have been developed and are being used by researchers to evaluate the performance of communication systems and to simulate channel characteristics when designing air interfaces or new wireless technologies across the network stack [80], [122]-[124].

This paper has provided a comprehensive overview of emerging $5 \mathrm{G}$ mmWave wireless system concepts, and has provided a compilation of important mmWave radio propagation models developed throughout the world to date. The paper demonstrates early standards work and illustrates the various models obtained by several independent groups based on extensive measurements and ray tracing methods at mmWave frequency bands in various scenarios.

The development of proper propagation models is vital, not only for the long-term development of future mmWave wireless systems but also for fundamental understanding by future engineers and students who will learn about and improve the nascent mmWave mobile industry that is just now being developed. Various companies have started 5G field trials, and some of them have achieved $20 \mathrm{Gbps}$ date rates [125], [126]. The fundamental information on path loss and shadowing surveyed in this paper is a prerequisite for moving further along the road to $5 \mathrm{G}$ at the unprecedented mmWave frequency bands.

\section{REFERENCES}

[1] J. Gubbi et al., "Internet of Things (IoT): A vision, architectural elements, and future directions," Future Generation Computer Systems, vol. 29, no. 7, pp. 1645-1660, Sept. 2013.
[2] T. S. Rappaport, "Spectrum Frontiers: The New World of MillimeterWave Mobile Communication," Invited keynote presentation, The Federal Communications Commission (FCC) Headquarters, Mar. 102016.

[3] T. S. Rappaport et al., "Millimeter Wave Mobile Communications for 5G Cellular: It Will Work!" IEEE Access, vol. 1, pp. 335-349, May 2013.

[4] S. Rangan, T. S. Rappaport, and E. Erkip, "Millimeter-wave cellular wireless networks: Potentials and challenges," Proceedings of the IEEE, vol. 102, no. 3, pp. 366-385, Mar. 2014.

[5] A. Ghosh et al., "Millimeter-wave enhanced local area systems: A high-datarate approach for future wireless networks," IEEE Journal on Selected Areas in Communications, vol. 32, no. 6, pp. 1152-1163, June 2014.

[6] W. Roh et al., "Millimeter-wave beamforming as an enabling technology for $5 \mathrm{G}$ cellular communications: theoretical feasibility and prototype results," IEEE Communications Magazine, vol. 52, no. 2, pp. 106-113, Feb. 2014.

[7] Federal Communications Commission, "Spectrum Frontiers Report and Order and Further Notice of Proposed Rulemaking: FCC16-89," July 2016. [Online]. Available: https://apps.fcc.gov/edocs_public/attachmatch/ FCC-16-89A1_Rcd.pdf

[8] S. Singh et al., "Tractable model for rate in self-backhauled millimeter wave cellular networks," IEEE Journal on Selected Areas in Communications, vol. 33, no. 10, pp. 2196-2211, Oct. 2015.

[9] K. Sundaresan et al., "Fluidnet: a flexible cloud-based radio access network for small cells," IEEE/ACM Transactions on Networking, vol. 24, no. 2, pp. 915-928, Apr. 2016

[10] P. Banelli et al., "Modulation formats and waveforms for 5G networks: Who will be the heir of OFDM?: An overview of alternative modulation schemes for improved spectral efficiency," IEEE Signal Processing Magazine, vol. 31, no. 6, pp. 80-93, Nov. 2014.

[11] N. Michailow et al., "Generalized frequency division multiplexing for 5th generation cellular networks," IEEE Transactions on Communications, vol. 62, no. 9, pp. 3045-3061, Sept. 2014.

[12] 5GCM, "5G Channel Model for bands up to $100 \mathrm{GHz}$," Tech. Rep., Oct. 2016. [Online]. Available: http://www.5gworkshops.com/5GCM.html

[13] K. Haneda et al., "Indoor 5G 3GPP-like channel models for office and shopping mall environments," in 2016 IEEE International Conference on Communications Workshops (ICC), May 2016, pp. 694-699.

[14] S. Deng, M. K. Samimi, and T. S. Rappaport, " $28 \mathrm{GHz}$ and $73 \mathrm{GHz}$ millimeter-wave indoor propagation measurements and path loss models," in IEEE International Conference on Communications Workshops (ICCW), June 2015, pp. 1244-1250.

[15] T. S. Rappaport and S. Deng, " $73 \mathrm{GHz}$ wideband millimeter-wave foliage and ground reflection measurements and models," in 2015 IEEE International Conference on Communication Workshop (ICCW), June 2015, pp. 12381243.

[16] K. Haneda et al., "5G 3GPP-like channel models for outdoor urban microcellular and macrocellular environments," in 2016 IEEE 83rd Vehicular Technology Conference (VTC 2016-Spring), May 2016, pp. 1-7.

[17] S. Nie et al., "72 GHz millimeter wave indoor measurements for wireless and backhaul communications," in 2013 IEEE 24th International Symposium on Personal Indoor and Mobile Radio Communications (PIMRC), Sept. 2013, pp. 2429-2433.

[18] K. Haneda et al., "Frequency-agile pathloss models for urban street canyons," IEEE Transactions on Antennas and Propagation, vol. 64, no. 5, pp. 19411951, May 2016.

[19] G. R. MacCartney and T. S. Rappaport, "Rural macrocell path loss models for millimeter wave wireless communications," IEEE Journal on Selected Areas in Communications, vol. 35, no. 7, pp. 1663-1677, July 2017.

[20] T. S. Rappaport et al., "Wideband millimeter-wave propagation measurements and channel models for future wireless communication system design," IEEE Transactions on Communications, vol. 63, no. 9, pp. 3029-3056, Sept. 2015.

[21] G. R. MacCartney, Jr. et al., "Indoor office wideband millimeter-wave propagation measurements and models at $28 \mathrm{GHz}$ and $73 \mathrm{GHz}$ for ultradense 5G wireless networks," IEEE Access, pp. 2388-2424, Oct. 2015.

[22] — , "Path loss models for 5G millimeter wave propagation channels in urban microcells," in 2013 IEEE Global Communications Conference (GLOBECOM), Dec. 2013, pp. 3948-3953.

[23] M. K. Samimi, T. S. Rappaport, and G. R. MacCartney, Jr., "Probabilistic omnidirectional path loss models for millimeter-wave outdoor communica- 
tions," IEEE Wireless Communications Letters, vol. 4, no. 4, pp. 357-360, Aug. 2015

[24] G. R. MacCartney, Jr. et al., "Millimeter wave wireless communications: New results for rural connectivity," in All Things Cellular'16: Workshop on All Things Cellular Proceedings, in conjunction with ACM MobiCom, Oct. 2016, pp. 31-36.

[25] S. Sun, G. R. MacCartney, Jr., and T. S. Rappaport, "Millimeter-wave distance-dependent large-scale propagation measurements and path loss models for outdoor and indoor 5G systems," in 2016 IEEE 10th European Conference on Antennas and Propagation (EuCAP), Apr. 2016, pp. 1-5.

[26] G. R. MacCartney, Jr., M. K. Samimi, and T. S. Rappaport, "Exploiting directionality for millimeter-wave wireless system improvement," in 2015 IEEE International Conference on Communications (ICC), June 2015, pp. 2416-2422.

[27] T. A. Thomas et al., "A prediction study of path loss models from 2-73.5 GHz in an urban-macro environment," in 2016 IEEE 83rd Vehicular Technology Conference (VTC 2016-Spring), May 2016, pp. 1-5.

[28] S. Sun et al., "Investigation of prediction accuracy, sensitivity, and parameter stability of large-scale propagation path loss models for $5 \mathrm{G}$ wireless communications," IEEE Transactions on Vehicular Technology, vol. 65, no. 5, pp. 2843-2860, May 2016.

[29] M. K. Samimi and T. S. Rappaport, "3-D millimeter-wave statistical channel model for 5G wireless system design," IEEE Transactions on Microwave Theory and Techniques, vol. 64, no. 7, pp. 2207-2225, July 2016.

[30] S. Hur et al., "Synchronous channel sounder using horn antenna and indoor measurements on $28 \mathrm{GHz}$," in 2014 IEEE International Black Sea Conference on Communications and Networking (BlackSeaCom), May 2014, pp. 83-87.

[31] T. S. Rappaport et al., "Broadband millimeter-wave propagation measurements and models using adaptive-beam antennas for outdoor urban cellular communications," IEEE Transactions on Antennas and Propagation, vol. 61, no. 4, pp. 1850-1859, Apr. 2013.

[32] O. H. Koymen et al., "Indoor mm-Wave Channel Measurements: Comparative Study of $2.9 \mathrm{GHz}$ and $29 \mathrm{GHz}$," in 2015 IEEE Global Telecommunications Conference Workshops (Globecom Workshops), Dec. 2015, pp. 1-6.

[33] G. P. Fettweis, "The tactile internet: applications and challenges," IEEE Vehicular Technology Magazine, vol. 9, no. 1, pp. 64-70, Mar. 2014.

[34] C. F. Mecklenbrauker et al., "Vehicular channel characterization and its implications for wireless system design and performance," Proceedings of the IEEE, vol. 99, no. 7, pp. 1189-1212, July 2011.

[35] J. Gozálvez, M. Sepulcre, and R. Bauza, "IEEE 802.11 p vehicle to infrastructure communications in urban environments," IEEE Communications Magazine, vol. 50, no. 5, pp. 176-183, May 2012.

[36] N. Bhushan et al., "Network densification: the dominant theme for wireless evolution into 5G," IEEE Communications Magazine, vol. 52, no. 2, pp. 82-89, Feb. 2014.

[37] A. Maeder et al., "The challenge of M2M communications for the cellular radio access network," in Würzburg Workshop on IP: Joint ITG and Euro-NF Workshop Visions of Future Generation Networks(EuroView), Aug. 2011, pp. $1-2$.

[38] A. Nikravesh et al., "An in-depth understanding of multipath tcp on mobile devices: measurement and system design," in Proceedings of the 22nd Annual International Conference on Mobile Computing and Networking, Oct. 2016, pp. 189-201.

[39] S. Deng et al., "WiFi, LTE, or both?: Measuring multi-homed wireless internet performance," in Proceedings of the 2014 Conference on Internet Measurement Conference, Nov. 2014, pp. 181-194.

[40] J. G. Andrews et al., "What will 5G be?" IEEE Journal on Selected Areas in Communications, vol. 32, no. 6, pp. 1065-1082, June 2014.

[41] E. Bastug, M. Bennis, and M. Debbah, "Living on the edge: The role of proactive caching in 5G wireless networks," IEEE Communications Magazine, vol. 52, no. 8, pp. 82-89, Aug 2014.

[42] N. Carapellese et al., "An energy consumption comparison of different mobile backhaul and fronthaul optical access architectures," in The European Conference on Optical Communication (ECOC), Sept. 2014, pp. 1-3.

[43] S. Hur et al., "Millimeter wave beamforming for wireless backhaul and access in small cell networks," IEEE Transactions on Communications, vol. 61, no. 10 , pp. 4391-4403, Oct. 2013.

[44] H2020 Project 5G-XHaul, "Dynamically Reconfigurable Optical-Wireless Backhaul/Fronthaul with Cognitive Control Plane for Small Cells and Cloud-RANs," 2015. [Online]. Available: http://www.5g-xhaul-project.eu/ index.html

[45] V. Chandrasekhar, J. G. Andrews, and A. Gatherer, "Femtocell networks: a survey," IEEE Communications Magazine, vol. 46, no. 9, pp. 59-67, Sept. 2008.

[46] M. Dohler et al., "Is the PHY layer dead?" IEEE Communications Magazine, vol. 49, no. 4, pp. 159-165, Apr. 2011.

[47] C.-X. Wang et al., "Cellular architecture and key technologies for 5G wireless communication networks," IEEE Communications Magazine, vol. 52, no. 2, pp. 122-130, Feb. 2014.

[48] X. Ge et al., "5G wireless backhaul networks: challenges and research advances," IEEE Network, vol. 28, no. 6, pp. 6-11, Nov. 2014.
[49] G. R. MacCartney and T. S. Rappaport, "73 ghz millimeter wave propagation measurements for outdoor urban mobile and backhaul communications in new york city," in 2014 IEEE International Conference on Communications (ICC), June 2014, pp. 4862-4867.

[50] J. N. Murdock and T. S. Rappaport, "Consumption factor and powerefficiency factor: A theory for evaluating the energy efficiency of cascaded communication systems," IEEE Journal on Selected Areas in Communications, vol. 32, no. 2, pp. 221-236, Feb. 2014.

[51] F. Haider et al., "Spectral efficiency analysis of mobile femtocell based cellular systems," in 2011 IEEE 13th International Conference on Communication Technology (ICCT), Sept. 2011, pp. 347-351.

[52] E. Hossain et al., "Evolution toward 5G multi-tier cellular wireless networks: An interference management perspective," IEEE Wireless Communications, vol. 21, no. 3, pp. 118-127, June 2014.

[53] J. G. Andrews et al., "An overview of load balancing in hetnets: old myths and open problems," IEEE Wireless Communications, vol. 21, no. 2, pp. 18-25, Apr. 2014.

[54] M. N. Tehrani, M. Uysal, and H. Yanikomeroglu, "Device-to-device communication in 5G cellular networks: challenges, solutions, and future directions," IEEE Communications Magazine, vol. 52, no. 5, pp. 86-92, May 2014.

[55] M. Yang et al., "Software-defined and virtualized future mobile and wireless networks: A survey," Mobile Networks and Applications, vol. 20, no. 1, pp. 4-18, Sept. 2015.

[56] P. K. Agyapong et al., "Design considerations for a 5g network architecture," IEEE Communications Magazine, vol. 52, no. 11, pp. 65-75, Nov. 2014.

[57] J. Van De Beek and F. Berggren, "Out-of-band power suppression in OFDM," IEEE communications letters, vol. 12, no. 9, pp. 609-611, Sept. 2008.

[58] A. Monk et al., "OTFS-Orthogonal Time Frequency Space," Computing Research Repository (CoRR), vol. abs/1608.02993, Aug. 2016.

[59] S. Sun et al., "MIMO for millimeter-wave wireless communications: beamforming, spatial multiplexing, or both?" IEEE Communications Magazine, vol. 52, no. 12, pp. 110-121, 2014.

[60] K. Haneda, "Channel models and beamforming at millimeter-wave frequency bands," IEICE Transactions on Communications, vol. 98, no. 5, pp. 755-772, May 2015.

[61] T. S. Rappaport et al., Millimeter Wave Wireless Communications. Pearson/Prentice Hall, 2015.

[62] T. Yamada et al., "Experimental evaluation of ieee 802.11ad millimeter-wave wlan devices," in 2015 21st Asia-Pacific Conference on Communications (APCC), Oct. 2015, pp. 278-282.

[63] A. Siligaris et al., "A 65-nm CMOS fully integrated transceiver module for 60-GHz Wireless HD applications," IEEE Journal of Solid-State Circuits, vol. 46, no. 12, pp. 3005-3017, Dec. 2011.

[64] E. Charfi, L. Chaari, and L. Kamoun, "PHY/MAC enhancements and qos mechanisms for very high throughput WLANs: A survey," IEEE Communications Surveys Tutorials, vol. 15, no. 4, pp. 1714-1735, Apr. 2013.

[65] E. Perahia and R. Stacey, Next Generation Wireless LANS: $802.11 n$ and 802.11 ac. Cambridge university press, 2013.

[66] L. Verma et al., "Wifi on steroids: 802.11 ac and 802.11 ad," IEEE Wireless Communications, vol. 20, no. 6, pp. 30-35, Dec. 2013.

[67] E. Perahia and M. X. Gong, "Gigabit wireless LANs: an overview of IEEE 802.11 ac and 802.11 ad," ACM SIGMOBILE Mobile Computing and Communications Review, vol. 15, no. 3, pp. 23-33, Nov. 2011.

[68] E. Perahia et al., "IEEE 802.11ad: Defining the next generation multiGbps Wi-Fi," in 2010 7th IEEE Consumer Communications and Networking Conference, Jan. 2010, pp. 1-5.

[69] A. Maltsev et al., "Channel Models for $60 \mathrm{GHz}$ WLAN Systems," Tech. Rep. doc.: IEEE 802.11-09/0334r8, May 2010. [Online]. Available: https://mentor.ieee.org/802.11/dcn/13/ 11-13-0794-00-00aj-channel-models-for-45-ghz-wlan-systems.docx

[70] -, "Channel modeling in the next generation mmWave Wi-Fi: IEEE 802.11ay standard," in European Wireless 2016; 22th European Wireless Conference, May 2016, pp. 1-8.

[71] IEEE 802.11 Working Group et al., "IEEE Standard for Information technology-Telecommunications and information exchange between systemsLocal and metropolitan area networks-Specific requirements Part 11: Wireless LAN Medium Access Control (MAC) and Physical Layer (PHY) Specifications," IEEE Std, vol. 802, no. 11, Sept. 2010.

[72] H. Moustafa and Y. Zhang, Vehicular networks: techniques, standards, and applications. Auerbach publications, 2009.

[73] E. Ben-Dor et al., "Millimeter-Wave $60 \mathrm{GHz}$ Outdoor and Vehicle AOA Propagation Measurements Using a Broadband Channel Sounder," in 2011 IEEE Global Telecommunications Conference - GLOBECOM 2011, Dec. 2011, pp. 1-6.

[74] T. S. Rappaport, S. DiPierro, and R. Akturan, "Analysis and simulation of adjacent service interference to vehicle-equipped digital wireless receivers from cellular mobile terminalss," in Vehicular Technology Conference Fall (VTC 2010-Fall), 2010 IEEE 72nd, Sept. 2010, pp. 1-5.

[75] T. S. Rappaport et al., "Small-Scale, Local Area, and Transitional Millimeter Wave Propagation for 5G Cellular Communications," IEEE Transactions on Antennas and Propagation, this issue. 
[76] V. Shivaldova et al., "On roadside unit antenna measurements for vehicleto-infrastructure communications," in Personal Indoor and Mobile Radio Communications (PIMRC), 2012 IEEE 23rd International Symposium on, Sept. 2012, pp. 1295-1299.

[77] D.-T. Phan-Huy, M. Sternad, and T. Svensson, "Making 5G adaptive antennas work for very fast moving vehicles," IEEE Intelligent Transportation Systems Magazine, vol. 7, no. 2, pp. 71-84, Summer 2015.

[78] T. S. Rappaport, J. N. Murdock, and F. Gutierrez, "State of the art in 60-GHz integrated circuits and systems for wireless communications," Proceedings of the IEEE, vol. 99, no. 8, pp. 1390-1436, Aug. 2011.

[79] ITU-R, "Attenuation by Atmospheric Gases," Tech. Rep. P.676-11, Sept. 2016.

[80] S. Sun, G. R. MacCartney, Jr., and T. S. Rappaport, "A Novel MillimeterWave channel simulator and applications for 5G wireless communications," in IEEE International Conference on Communication (ICC), May 2017, pp. $1-7$.

[81] H. Xu et al., "Measurements and models for $38-\mathrm{GHz}$ point-to-multipoint radiowave propagation," IEEE Journal on Selected Areas in Communications, vol. 18 , no. 3, pp. 310-321, Mar. 2000.

[82] ITU-R, "Specific attenuation model for rain for use in prediction methods, propagation in non-ionized media," Tech. Rep. P.838-3, 2005.

[83] S. Sun et al., "Propagation Path Loss Models for 5G Urban Micro- and Macro-Cellular Scenarios," in 2016 IEEE 83rd Vehicular Technology Conference (VTC 2016-Spring), May 2016, pp. 1-6.

[84] _ _ "Path loss, shadow fading, and line-of-sight probability models for $5 \mathrm{G}$ urban macro-cellular scenarios," in 2015 IEEE Globecom Workshops (GC Wkshps), Dec. 2015, pp. 1-7.

[85] H. T. Friis, "A note on a simple transmission formula," Proceedings of the IRE, vol. 34, no. 5, pp. 254-256, May 1946.

[86] I. Uchendu and J. R. Kelly, "Survey of beam steering techniques available for millimeter wave applications," Progress In Electromagnetics Research B, vol. 68, pp. 35-54, Mar. 2016.

[87] T. Nitsche et al., "Steering with eyes closed: mm-wave beam steering without in-band measurement," in Computer Communications (INFOCOM), 2015 IEEE Conference on, Apr. 2015, pp. 2416-2424.

[88] G. R. MacCartney, Jr. et al., "Millimeter-Wave Human Blockage at $73 \mathrm{GHz}$ with a Simple Double Knife-Edge Diffraction Model and Extension for Directional Antennas," in IEEE 84th Vehicular Technology Conference Fall (VTC 2016-Fall), Sept. 2016, pp. 1-6.

[89] I. Rodriguez et al., "Analysis of $38 \mathrm{GHz}$ mmwave propagation characteristics of urban scenarios," in European Wireless 2015; 21th European Wireless Conference; Proceedings of, May 2015, pp. 1-8.

[90] J. Ryan, G. R. MacCartney, Jr., and T. S. Rappaport, "Indoor Office Wideband Penetration Loss Measurements at $73 \mathrm{GHz}$," in IEEE International Conference on Communications Workship (ICCW), May 2017.

[91] M. Rumney, "Testing 5G: Time to throw away the cables," Microwave Journal, Nov. 2016.

[92] mmMAGIC, "Measurement results and final mmmagic channel models," Tech. Rep. H2020-ICT-671650-mmMAGIC/D2.2, May 2017. [Online]. Available: https://5g-mmmagic.eu/results/

[93] M. K. Samimi et al., " $28 \mathrm{GHz}$ millimeter-wave ultrawideband smallscale fading models in wireless channels," in 2016 IEEE 83rd Vehicular Technology Conference (VTC 2016-Spring), May 2016, pp. 1-6.

[94] M. K. Samimi and T. S. Rappaport, "Local multipath model parameters for generating $5 \mathrm{G}$ millimeter-wave 3GPP-like channel impulse response," in 2016 10th European Conference on Antennas and Propagation (EuCAP), Apr. 2016, pp. 1-5.

[95] S. Deng et al., "Indoor and Outdoor 5G Diffraction Measurements and Models at 10, 20, and $26 \mathrm{GHz}$," in 2016 IEEE Global Communications Conference (GLOBECOM), Dec. 2016, pp. 1-7.

[96] 3GPP, "Technical specification group radio access network; study on 3d channel model for lte (release 12)," 3rd Generation Partnership Project (3GPP), TR 36.873 V12.2.0, June 2015. [Online]. Available: http://www.3gpp.org/dynareport/36873.htm

[97] R. B. Ertel et al., "Overview of spatial channel models for antenna array communication systems," IEEE personal communications, vol. 5, no. 1, pp. 10-22, Feb. 1998

[98] S. Sun et al., "Millimeter wave small-scale spatial statistics in an urban microcell scenario," in IEEE International Conference on Communication (ICC), May 2017, pp. 1-7.

[99] T. S. Rappaport, S. Sun, and M. Shafi, "5G channel model with improved accuracy and efficiency in mmwave bands," IEEE 5G Tech Focus, Mar. 2017.

[100] _ "Investigation and comparison of 3GPP and NYUSIM channel models for 5G wireless communications," 2017 IEEE 86th Vehicular Technology Conference (VTC Fall), Sept. 2017.

[101] 3GPP, "Study on channel model for frequencies from 0.5 to $100 \mathrm{GHz}$," 3rd Generation Partnership Project (3GPP), TR 38.901 V14.0.0, May. 2017. [Online]. Available: http://www.3gpp.org/DynaReport/38901.htm

[102] METIS2020, "METIS Channel Model," Tech. Rep. METIS2020, Deliverable D1.4 v3, July 2015. [Online]. Available: https://www.metis2020.com/ wp-content/uploads/deliverables/METIS_D1.4_v3.0.pdf
[103] M. K. Samimi and T. S. Rappaport, "3-D statistical channel model for millimeter-wave outdoor mobile broadband communications," in IEEE International Conference on Communications (ICC), June 2015, pp. 2430-2436

[104] MiWeba, "WP5: Propagation, Antennas and Multi-Antenna Technique; D5.1: Channel Modeling and Characterization," Tech. Rep. MiWEBA Deliverable D5.1, June 2014. [Online]. Available: http://www.miweba.eu/ wp-content/uploads/2014/07/MiWEBAD5.1v1.011.pdf

[105] S. Hur et al., "Proposal on Millimeter-Wave Channel Modeling for 5G Cellular System," IEEE Journal of Selected Topics in Signal Processing, vol. 10, no. 3, pp. 454-469, Apr. 2016.

[106] International Telecommunications Union, "Guidelines for evaluation of radio interface technologies for IMT-Advanced," Geneva, Switzerland, REP. ITU-R M.2135-1, Dec. 2009

[107] J. Jarvelainen et al., "Evaluation of millimeter-wave line-of-sight probability with point cloud data," IEEE Wireless Communications Letters, vol. 5, no. 3, pp. 228-231, June 2016.

[108] S. Piersanti, L. A. Annoni, and D. Cassioli, "Millimeter waves channel measurements and path loss models," in 2012 IEEE International Conference on Communications (ICC), June 2012, pp. 4552-4556.

[109] E. Semaan et al., "Outdoor-to-indoor coverage in high frequency bands," in 2014 IEEE Globecom Workshops (GC Wkshps), Dec. 2014, pp. 393-398.

[110] G. R. MacCartney, Jr. et al., "Millimeter-wave omnidirectional path loss data for small cell 5G channel modeling," IEEE Access, vol. 3, pp. 1573-1580, Aug. 2015

[111] G. R. MacCartney, Jr. and T. S. Rappaport, "Study on 3GPP rural macrocell path loss models for millimeter wave wireless communications," in 2017 IEEE International Conference on Communications (ICC), May 2017, pp $1-7$.

[112] 3GPP, "Technical specification group radio access network; channel model for frequency spectrum above $6 \mathrm{GHz}$," 3rd Generation Partnership Project (3GPP), TR 38.900 V14.2.0, Dec. 2016. [Online]. Available: http://www.3gpp.org/DynaReport/38900.htm

[113] WINNER+ D5.3, "Final channel models," Tech. Rep. V1.0, CELTIC CP5026 WINNER+ project, 2010

[114] M. Hata, "Empirical formula for propagation loss in land mobile radio services," IEEE Transactions on Vehicular Technology, vol. 29, no. 3, pp 317-325, Aug. 1980.

[115] S. Sun et al., "Synthesizing Omnidirectional antenna patterns, received power and path loss from directional antennas for $5 \mathrm{G}$ millimeter-wave communications," in IEEE Global Communications Conference (GLOBECOM), Dec. 2015, pp. 3948-3953.

[116] G. R. MacCartney, M. K. Samimi, and T. S. Rappaport, "Omnidirectional path loss models in new york city at $28 \mathrm{ghz}$ and $73 \mathrm{ghz}$," in 2014 IEEE 25th Annual International Symposium on Personal, Indoor, and Mobile Radio Communication (PIMRC), Sept 2014, pp. 227-231.

[117] J. B. Andersen, T. S. Rappaport, and S. Yoshida, "Propagation measurements and models for wireless communications channels," IEEE Communications Magazine, vol. 33, no. 1, pp. 42-49, Jan. 1995.

[118] K. Bullington, "Radio propagation at frequencies above 30 megacycles," Proceedings of the IRE, vol. 35, no. 10, pp. 1122-1136, Oct. 1947.

[119] M. J. Feuerstein et al., "Path loss, delay spread, and outage models as functions of antenna height for microcellular system design," IEEE Transactions on Vehicular Technology, vol. 43, no. 3, pp. 487-498, Aug. 1994.

[120] 3GPP, "Correction for low and high frequency model harmonization," Ericsson, Samsung, NTT DOCOMO, Nokia, Intel, Telstra, Tech. Rep. TDOC R1-1701195, Jan. 2017.

[121] 3GPP, "New measurements at $24 \mathrm{GHz}$ in a rural macro environment," Telstra, Ericsson, Tech. Rep. TDOC R1-164975, May 2016.

[122] V. K. Rajendran et al., "Concepts and Implementation of a Semantic Web Archiving and Simulation System for RF Propagation Measurements," in Vehicular Technology Conference (VTC 2011-Fall), 2011 IEEE, Sept. 2011, pp. $1-5$.

[123] S. Jaeckel et al., "Quadriga: A 3-d multi-cell channel model with time evolution for enabling virtual field trials," IEEE Transactions on Antennas and Propagation, vol. 62, no. 6, pp. 3242-3256, Mar. 2014.

[124] New York University, NYUSIM, 2016. [Online]. Available: http://wireless. engineering.nyu.edu/nyusim/

[125] Huawei, "5G: Huawei and Vodafone achieve 20Gbps for single-user outdoor at E-Band," July 2016. [Online]. Available: http://www.huawei.com/en/ news/2016/7/huawei-vodafone-5g-test

[126] Ericsson, "Ericsson and Telstra conduct the first live 5G trial in Australia," Sept. 2016. [Online]. Available: https://www.ericsson.com/news/ 160920-ericsson-telstra-5g-trial-australia_244039854_c 